\title{
Beta-glucan-loaded nanofiber dressing improves wound healing in diabetic mice
}

Jostein Gripa,b , Rolf Einar Engstad ${ }^{\mathrm{a}}$, Ingrid Skjæveland ${ }^{\mathrm{a}}$, Nataša Škalko-Basnet ${ }^{\mathrm{b}}$, Johan Isaksson $^{\mathrm{c}}$, Purusotam Basnet ${ }^{\mathrm{d}, \mathrm{e}}$, Ann Mari Holsæter ${ }^{\mathrm{b}, *}$

${ }^{a}$ Biotec BetaGlucans AS, 9019 Tromsø, Norway

${ }^{b}$ Drug Transport and Delivery Research Group, Department of Pharmacy, Faculty of Health Sciences, University of Tromsø The Arctic University of Norway, 9037 Tromsø, Norway

${ }^{\mathrm{c}}$ Department of Chemistry, University of Tromsø The Arctic University of Norway, 9037

Tromsø, Norway

${ }^{\mathrm{d}}$ Women's Health and Perinatology Research Group, Department of Clinical Medicine, University of Tromsø The Arctic University of Norway, 9037 Tromsø, Norway

${ }^{\mathrm{e}}$ Department of Obstetrics and Gynecology, University Hospital of North Norway, Sykehusveien 5738, 9038 Tromsø, Norway.

\section{*Corresponding author}

Ann Mari Holsæter, PhD

E-mail address: ann-mari.holsater@uit.no

Telephone: +4777646719 


\begin{abstract}
The increased prevalence of chronic wounds requires novel treatment options. The aim of this study was to develop a beta-glucan $(\beta \mathrm{G})$-loaded nanofiber wound dressing. Nanofibers were prepared using the needle-free Nanospider ${ }^{\mathrm{TM}}$ technology, an electrospinning method which enables the production of nanofibers at an industrial scale. The $\beta \mathrm{G}$ was selected as active ingredient based on its confirmed wound healing potential in both animals and humans. Hydroxypropyl methylcellulose (HPMC) and polyethylene oxide (PEO) were included as copolymers. Rheological profiles of spinning solutions containing HPMC, PEO, $\beta \mathrm{G}$, ethanol and water, were optimized. The nanofiber formation was confirmed by Field Emission Scanning Electron Microscopy (FE-SEM), and both nanofibers with ( $\beta$ G-nanofibers) or without $\beta G$ (NoßG-nanofibers) were evaluated by their swelling index and FT-IR spectroscopy. The formulations, active ingredient and excipients, were tested for their possible in vitro toxicity in keratinocytes. Finally, the wound healing potential of the nanofibers was tested in externaly induced excisional wounds in male diabetic $\mathrm{db} / \mathrm{db}$ mice. Three different doses of $\beta G$-nanofibers and the $\beta G$-free, No $\beta G$-nanofibers, were evaluated for their in vivo wound healing efficacy. All nanofiber-treatments provided improved wound healing as compared to the negative control (water). All $\beta \mathrm{G}$-nanofiber-treated groups exhibited significantly improved wound healing as compared to the NoßG-nanofiber-treated group, indicating the potential of $\beta \mathrm{G}$-nanofibers as wound dressing.
\end{abstract}

Key words: beta-glucan; nanofiber; diabetic $\mathrm{db} / \mathrm{db}$ mice; needle-free electrospinning; wound dressing; wound healing 


\section{Introduction}

With an aging population and increased prevalence of impaired/chronic wounds, the search for optimal wound dressings has been intensified in the past years [1]. The optimal wound dressing should not only accelerate the wound healing process but also minimize the pain associated with wound healing and dressing change [2]. To develop a superior wound dressing, it is crucial to understand the complexity of the skin repair processes involving many different cell types, the role of signals molecules and feedback loops orchestrating the cellular response [3-5]. Delayed/impaired wound healing might be caused by different underlying pathologies, such as co-morbidities and peripheral vascular disease and biofilm formation [5,6]. Considering the challenges in wound treatment and related health cost [7], pharmaceutical and biotechnological industries, as well as academia, focus more on developing advanced and active wound dressings, to replace the non-active convential wound dressings [8].

Among emerging technologies in wound dressing development, nanofibers have gained an increased attention. The nanofibers closely resemble the native extracellular matrix, and, formed as fiber mats, offer improved physical and mechanical wound protection, even against bacterial invasions [9]. Moreover, the fibers permit gas exchange, facilitating the wound healing process. Drugs and various active substances can be incorporated within the fiber mats. By the optimization of the spinning conditions, the release of nanofiber-associated drugs can be modified and controlled [10,11]. Electrospinning is the most widely used nanofiber fabrication technique [12], as it offers high flexibility in production and enables controllable nanofiber pore structure from a wide variety of synthetic and natural polymers [13].

Among various active substances with beneficial effects on wound healing, we have focused on $\beta$-glucans $(\beta G)$. They are known to re-activate/activate the innate immune system by binding to the dectin-1 receptor on macrophages, dendritic cells and neutrophils [14]. This activation can help to revert immunosuppressed macrophages into a responsive phenotype [15], and thus improve the stalled wound healing. A formulation containing $\beta G$ is currently on the market as a hydrogel for treatment of dry chronic wounds [16,17]. The soluble $2.5 \%(\mathrm{w} / \mathrm{v})$ $\beta G$ used in this formulation, as well as in this current study, is a $\beta-1,3 / 1,6$ glucan isolated from cell walls of Baker's yeast (Saccharomyces cerevisiae), and further processed by a patented solubilizing procedure [18]. This soluble $\beta \mathrm{G}$ forms a gel at room temperature, and 
has, in previous studies, been confirmed to maintain its immune-stimulating activity both in human cell-lines and animal models [19,20].

The main aim of this study was to develop an active nanofiber dressing which could be clinically relevant and effective in the treatment of medium exuding chronic wounds. Moreover, we targeted a nanofiber production method applicable in an industrial scale manufacturing. For these reasons, the needle-free Nanospider ${ }^{\mathrm{TM}}$ spinning technology, developed by Elmarco (Liberec, Czech Republic), was selected. This technology enables a 26-80 times faster production of nanofibers than the traditional single needle electrospinning setup, even when using the smallest Nanospider ${ }^{\mathrm{TM}}$ Lab machine, with a production speed of around $40 \mathrm{~mL} / \mathrm{h}[21,22]$. We selected hydroxypropyl methyl cellulose (HPMC) as a copolymer since cellulose derivatives are known to form homogenous nanofiber mats of uniform quality [23]; and polyethylene oxide (PEO) was added to assure fiber formation. The $\beta G$-loaded nanofibers were optimized and evaluated in the chronic wound model, diabetic $\mathrm{db} / \mathrm{db}$ mice.

\section{Materials and methods}

\subsection{Materials}

Milli-Q water was produced using the Q-Pod® by Merck Millipore (Billerica, MA, USA). Sodium chloride and calcium chloride was purchased from Merck (Kenilworth, USA). Soluble beta-1,3/1,6-glucan ( $\beta \mathrm{G} ; 2.5 \% \mathrm{w} / \mathrm{w})$ was a gift from Biotec BetaGlucans AS (Tromsø, Norway). The two types of hydroxypropyl methylcellulose (HPMC) used in this study, Metolose 60SH-4000 (Hypromellose 2910) and Metolose 90SH-4000 (Hypromellose 2208), were generous gifts from HARKE Pharma GmbH (Mülheim an der Ruhr, Germany). Polyethylene oxide (PEO) was obtained from Sigma-Aldrich (Taufkirchen, Germany). Ethanol (EtOH, 96\% v/v) was purchased from Kemetyl (Kolbotn, Norway).

\subsection{Polymer solution preparation and characterization}

Five different polymer spinning solutions were prepared (Table 1). Two solutions were applied in the production of the final electrospun nanofibers and used in the in vivo studies ( $\beta \mathrm{G}-\mathrm{Sol}$ and No $\beta \mathrm{G}-\mathrm{Sol}$ ), and the three other solutions were considered the reference solutions (Ref-Sol 1, 2 and 3). All Ref-Sol were prepared as described by Frenot and co-workers [23], 
who successfully produced HPMC fibers from similar solutions using the traditional needle electrospinning setup. Two different HPMC qualities were tested (HPMC1 and HMPC2), and their specifications are given in Table 2 together with the properties of the other polymers applied in the formulations, namely PEO and $\beta \mathrm{G}$. The solvents applied were EtOH alone (NoßG-Sol) or EtOH in combination with water ( $\beta$ G-Sol, Ref-Sol 1, Ref-Sol 2, Ref-Sol 3). PEO, HPMC and $\beta G$ were mixed from pre-made solutions. The PEO solution $(5 \%, \mathrm{w} / \mathrm{w})$ was prepared in $\mathrm{EtOH}(96 \%)$ and heated at $70{ }^{\circ} \mathrm{C}$ under constant stirring for 30 minutes, before cooling to room temperature [24]. The HPMC1 ethanolic solution, premade for the $\beta \mathrm{G}$-Sol and NoßG-Sol, had a final HPMC concentration of $2.91 \%(\mathrm{w} / \mathrm{w})$, and was prepared at room temperature, whereas the premade HPMC solutions made for the Ref-Sols, containing either HPMC1 or HMPC2, were made from EtOH: water $(50 / 50, \mathrm{v} / \mathrm{v})$ solvent blends. The $\beta \mathrm{G}-\mathrm{Sol}$ was prepared by heating the gelled $\beta \mathrm{G}(2.5 \%, \mathrm{w} / \mathrm{w})$ to $60{ }^{\circ} \mathrm{C}$. Heating was conducted to break up aggregates and convert the gel into the liquid form. The HPMC-Sol, PEO-Sol and $\beta$ G-Sol were mixed to reach the final polymer compositions of the spinning solutions, as listed in Table 1.

Table 1. Composition of the solutions used in electrospinning.

\begin{tabular}{|c|c|c|c|c|c|c|c|}
\hline \multirow[b]{2}{*}{ Solution } & \multirow{2}{*}{$\begin{array}{c}\boldsymbol{\beta G} \\
(\%, \mathrm{w} / \mathrm{w})\end{array}$} & \multicolumn{2}{|c|}{ HРMC $(\%, w / w)$} & \multirow{2}{*}{$\begin{array}{c}\text { PEO } \\
(\%, w / w)\end{array}$} & \multirow{2}{*}{$\begin{array}{c}\text { EtOH } \\
(\%, w / w)\end{array}$} & \multirow{2}{*}{$\begin{array}{c}\mathbf{H}_{2} \mathbf{O} \\
(\%, \mathrm{w} / \mathrm{w})\end{array}$} & \multirow{2}{*}{$\begin{array}{l}\text { Total dry } \\
\text { content } \\
(\%, w / w)\end{array}$} \\
\hline & & $\begin{array}{c}\text { HPMC1 } \\
*\end{array}$ & $\begin{array}{c}\text { HPMC2 } \\
*\end{array}$ & & & & \\
\hline$\beta \mathrm{G}-\mathrm{Sol}$ & 0.74 & 1.97 & & 0.30 & 68.15 & $28.84 * *$ & 3.01 \\
\hline No $\beta G-S o l$ & & 2.80 & & 0.43 & 96.78 & - & 3.22 \\
\hline Ref-Sol 1 & & 2.86 & & & 45.14 & 52.00 & 2.86 \\
\hline Ref-Sol 2 & & & 2.86 & & 45.14 & 52.00 & 2.86 \\
\hline Ref-Sol 3 & & & 2.14 & & 45.47 & 52.39 & 2.14 \\
\hline
\end{tabular}

* Specification given in Table 2.

**The water content in $\beta \mathrm{G}-\mathrm{Sol}$ originates from the $2.5 \%(\mathrm{w} / \mathrm{w}) \beta \mathrm{G}$.

Table 2. Characteristics of the polymers used for the spinning solutions.

\begin{tabular}{|cccccc|}
\hline $\begin{array}{c}\text { Trade } \\
\text { name }\end{array}$ & $\begin{array}{c}\text { Abbre- } \\
\text { viations }\end{array}$ & $\begin{array}{c}\text { Approx. Mw } \\
\text { (kDa) }\end{array}$ & $\begin{array}{c}\text { Substitution } \\
\text { type }\end{array}$ & $\begin{array}{c}\text { Methoxy } \\
\text { content (\%) }\end{array}$ & $\begin{array}{c}\text { Hydroxypropoxy } \\
\text { content (\%) }\end{array}$ \\
60 SH4000 & HPMC1 & 270 & 2910 & $28.0-30.0$ & $7.0-12.0$ \\
90 SH4000 & HPMC2 & 270 & 2208 & $19.0-24.0$ & $4.0-12.0$ \\
& PEO & 900 & & n.a. & n.a. \\
SBG & $\beta G$ & $300-1500$ & & n.a. & n.a. \\
\hline
\end{tabular}


The viscosities of the electrospinning solutions were measured and compared using a Discovery HR-2 Hybrid Rheometer (TA Instruments, New Castle, DE, USA) equipped with $40 \mathrm{~mm}$ crosshatched plate geometry. The crosshatched plate was lower to form a gap of 1050 $\mu \mathrm{m}$ against the platform, excess solution removed and the plate lowered to operational height of $1000 \mu \mathrm{m}$. The samples were soaked for $120 \mathrm{sec}$ at $25^{\circ} \mathrm{C}$ to provide a stable temperature during the measurement. The flow sweep protocol was performed as a logarithmic sweep from 10 to $1000 \mathrm{~Pa}$, logging 10 point per decade using a steady state sensing. The results were processed by the Trios software v. 3.2.0.3877 (TA Instruments, New Castle, DE, USA) to determine the flow characteristics using the viscosity as a function of the shear rate.

\subsection{Electrospinning}

The Elmarco Nanospider ${ }^{\mathrm{TM}}$ Lab electrospinning machine (Liberec, Czech Republic), with a roll-to-roll collection unit and an adjustable substrate speed of between $0-5000 \mathrm{~mm} / \mathrm{min}$, and a spinning electrode wire with a power supply capable of delivering $80 \mathrm{kV}$ DC current, was applied. The ambient room temperature was kept at $26 \pm 1^{\circ} \mathrm{C}$, with a relative humidity of 35 $\pm 2 \%$. The distance from the electrode to the collector was $20 \mathrm{~cm}$, and the maximum voltage of $80 \mathrm{kV}$ was chosen. The polymer solution carriage reservoir, feeding the electrode with the polymer solution, was set to move at max speed, corresponding to $300 \mathrm{~mm} / \mathrm{s}$; the substrate setting applied was $2-4 \mathrm{~mm} / \mathrm{min}$, depending on the targeted thickness (weight/area) of the nanofiber.

\subsection{Fourier-Transform Infrared Spectroscopy (FTIR) analysis}

The dry polymer materials included in the electrospun nanofibers (PEO, HMPC, and freeze dried $\beta G$ ), and the final electrospun nanofibers ( $\beta G$ - and NoßG-nanofibers) were analyzed on a Cary 630 FTIR instrument from Agilent Technologies (Santa Clara, CA, USA); equipped with a diamond ATR sampling accessory. All FTIR spectra were acquired in 64 scans.

\subsection{Field Emission Scanning Electron Microscopy (FE-SEM) analysis}

The morphology of the electrospun fibers was investigated by Field Emission Scanning

Electron Microscopy (FE-SEM), applying the Zeiss Sigma FE-SEM (Carl Zeiss, Oberkochen, 
Germany). The samples were put on stubs with double sided carbon tape and two drops of conductive silver paint (Agar Scientific, Essex, UK) added to increase conductivity. The samples were dried for $24 \mathrm{~h}$ under an incandescent lamp, before they were sputter-coated with gold/palladium using a Polaron SC7640 (Quorum Technologies LTD, Kent, UK).

Micrographs were taken from the middle of the product roll, to assure a representative sample for imaging.

\subsection{Swelling index determination}

The absorption capacity of the two types of nanofibers applied in the animal study were investigated using a modified method by Boateng and co-workers [25]. The absorption fluid used in testing was the "Solution A" from the ISO standard EN 13726-1 [26] which simulates the ions concentration in human wound exudate $\left(142 \mathrm{mmol} \mathrm{Na}^{2+}, 2.5 \mathrm{mmol} \mathrm{Ca}^{2+}\right)$. The fiber mats were cut into $8 \times 8 \mathrm{~cm}$ pieces. The dry fibers were placed in $140 \mathrm{~mm} \varnothing$ Petri dishes (VWR, Norway), weighed ( $\mathrm{W}_{\mathrm{d}}$ ), and then submerged in "Solution A" for one minute (Sup.

Figure 1). The excess liquid was immediately removed and the Petri dish left at a $90^{\circ}$ angle to drain liquid from the fibers for one minute. The excess liquid was removed using tissue paper before the weight of the wet fiber mats $\left(\mathrm{W}_{\mathrm{s}}\right)$ was determined. The experiment was done in triplicates and the swelling index $\mathrm{I}_{\mathrm{S}}(\%)$ was calculated using Equation 1.

\section{Equation 1}

$$
I_{s}(\%)=\frac{W_{s}-W_{d}}{W_{d}} \times 100
$$

$\mathrm{W}_{\mathrm{d}}=$ Weight of dry film

$\mathrm{W}_{\mathrm{s}}=$ Weight of film after swelling

\subsection{Cytotoxicity of electrospun nanofibers}

The in vitro toxicity of the electrospun nanofibers was evaluated on keratinocytes (HaCaT cells) supplied from Thermo Fisher Scientific (Waltham, USA). Cells were cultured in RPMI medium (Sigma Aldrich, Steinheim, Germany) supplemented with glutamine and $10 \%$ fetal calf serum (FCS) at humidified $5 \% \mathrm{CO}_{2}$ and $37{ }^{\circ} \mathrm{C}$. The toxicity of the electrospun nanofibers 
was tested by the MTT assay using the cell proliferation kits from Roche (Sigma Aldrich) according to the instruction provided by the supplier. In brief, $90 \mu \mathrm{L}$ of trypsinized $\mathrm{HaCaT}$ cells were plated in the flat bottom 96 wells plate as the cell concentration $5 \times 10^{5}$ cells $/ \mathrm{mL}$. To each well $10 \mu \mathrm{L}$ media only (control) or the samples diluted in media (resulting in a final concentrations of 1,10 or $100 \mu \mathrm{g} / \mathrm{mL}$, respectively) were added. The cells were incubated for 24 hours at humidified $5 \% \mathrm{CO}_{2}$ and $37{ }^{\circ} \mathrm{C}$. The next day, $10 \mu \mathrm{L}$ of MTT (final concentration of $0.5 \mathrm{mg} / \mathrm{mL}$ ) was added to each well and the cells incubated for 4 hours for the development of violet crystals of formazon. After 4 hours, $100 \mu \mathrm{L}$ of solubilizing reagents (supplied in the assay kits) was added to each well and the cells were kept in the humidified incubator for 24 hours. The UV absorption of soluble formazon was determined on ELISA plate reader at 580 $\mathrm{nm}$. The UV absorption determined for the control group was considered as $100 \%$ viable and the effects of various concentration of tested samples on cell toxicity were expressed as percentage viability as compared to the control. Results are expressed as the mean of two independent experiments for each sample.

\subsection{Effect of nanofibers on wound healing in diabetic mice}

\subsubsection{Animal study setup}

The in vivo evaluation of wound healing potential of nanofibers was carried out following the protocol previously described by our group [27]. The study was conducted in accordance to the Home Office Regulations UK and the specific requirements of diabetic animals [28]. Male diabetic db/db mice (BKS.Cg-m Dock7m +/+ Leprdb /J mice), 9-10 weeks old (weight $45.6 \pm$ $2.6 \mathrm{~g}$ ) provided by Jackson Labs (Bar Harbour, ME, USA) were randomly divided into six experimental groups (10 mice per group); four groups were treated with nanofibers and two groups received the positive and negative control, respectively. Three different doses of $\beta \mathrm{G}$ nanofibers and NoßG-nanofibers (vehicle control) were tested (Table 3). The treatment was applied on day 0,4 and 8 in the nanofiber treated groups, and on day 0,2, 4, 6 and 8 for the animals receiving negative control, and on day $0,1,2,3,4,5$ and 6 for the animals treated with positive controls (more details are given in supplement; Sup. Table 1). 
Table 3. The doses of $\beta \mathrm{G}$-nanofibers applied in the in vivo study on diabetic $\mathrm{db} / \mathrm{db}$ mice wound model.

\begin{tabular}{|cccc|}
\hline Treatment group & Nanofiber weigh $\mathbf{( m g / \mathbf { c m } ^ { 2 } )}$ & Layers & 及G dose $\mathbf{( m g )}$ \\
\hline Low $\beta \mathrm{G}$ & 0.77 & 1 & 0.19 \\
Medium $\beta \mathrm{G}$ & 1.50 & 1 & 0.37 \\
High $\beta \mathrm{G}$ & 4.02 & 3 & 0.99 \\
\hline No $\beta \mathrm{G}$ & 2.10 & 1 & 0 \\
\hline
\end{tabular}

\subsubsection{Wound induction and treatment}

The wounds were induced on day 0 . All animals were anaesthetized using $4 \%$ isoflurane (IsoFlo ${ }^{\circledR}$, purchased from Zoetis (London, UK)) and 96\%, air followed by $2 \%$ isoflurane and 98\% (v/v) air for maintenance. Their left dorsal flank was shaved and cleaned, and a single standardized full-thickness wound $(1 \mathrm{x} 1 \mathrm{~cm})$ created approximately $10 \mathrm{~mm}$ from the spine. The wounds were covered with a polyurethane occlusive film (Bioclusive ${ }^{\circledR}$ film, purchased from Systagenix Wound Management (Gargrave, UK)). Water for injection (50 $\mu \mathrm{L})$ was injected under the occlusion film of the animals in the negative control group. In the positive control group, mice received $50 \mu \mathrm{L}$ solution containing growth factors; $10 \mu \mathrm{g} /$ dose plateletderived growth factor-BB and $1 \mu \mathrm{g}$ /dose transforming growth factor-alpha (both purchased from PeproTech EC Ltd, London, UK) dissolved in 0.5\% (w/v) HPMC. For the nanofibertreated animals the occlusive film was applied over the nanofibers after they were placed onto the wound forming a hydrogel (in situ), as shown in Figure 1. The nanofiber mats were cut in $1 \times 1 \mathrm{~cm}$ slices except for the group treated with High $\beta \mathrm{G}$ nanofibers; these nanofibers were cut in $1 \times 3 \mathrm{~cm}$ and folded twice to form three layers (same contact area). All animals received $75 \mu \mathrm{g} / \mathrm{kg}$ Vetergesic ${ }^{\circledR}$ (purchased from Alstoe Animal Health (Espoo, Finland)) post-surgery - dose volume $100 \mu \mathrm{L}$. When necessary, the Vetergesic ${ }^{\circledR}$ treatment was repeated every 12 hours. 


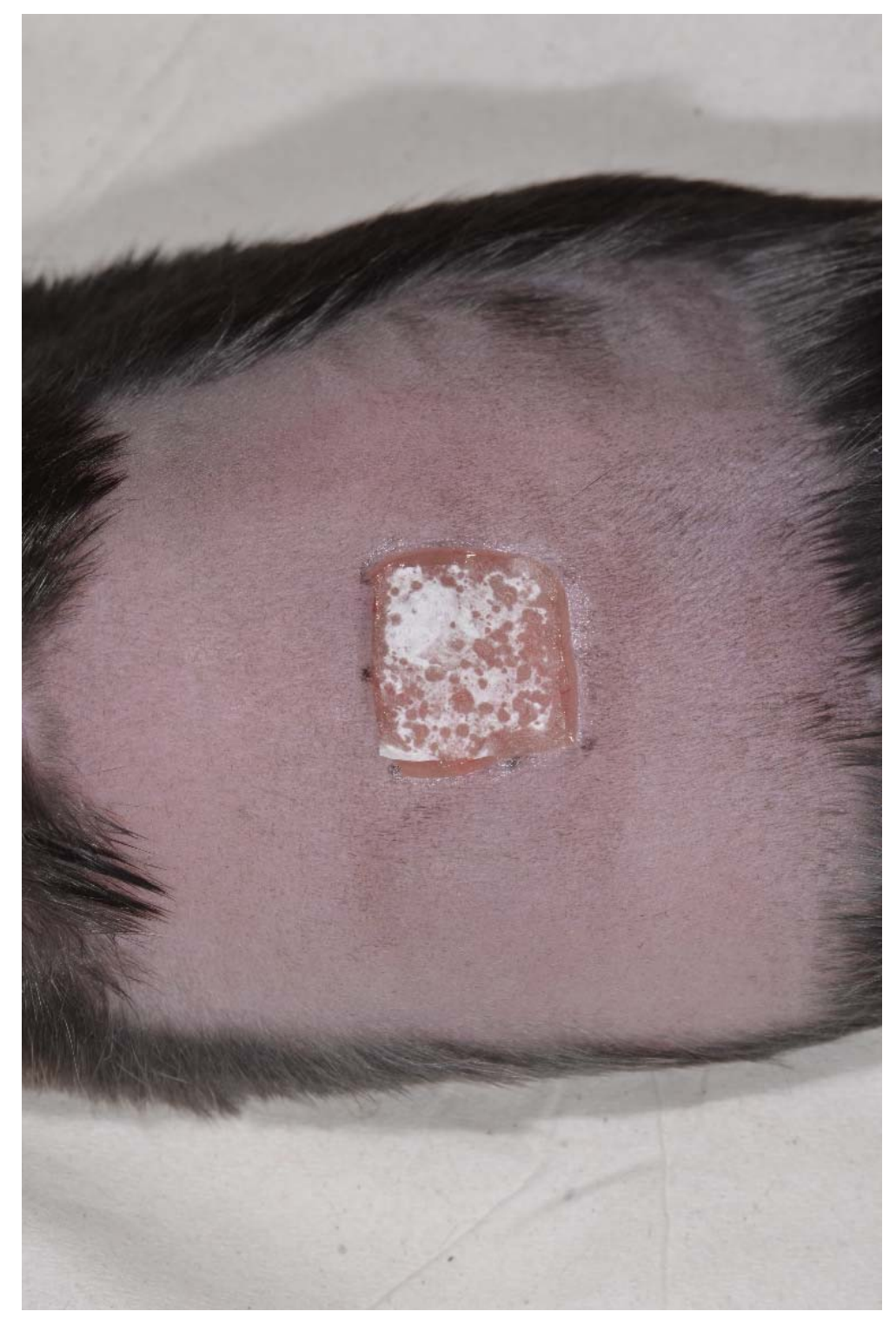

Figure 1. The in situ formed hydrogel (upon administration of nanofiber onto the wound) before covering with the polyurethane occlusive film.

\subsubsection{Wound closure assessment}

Image Pro Plus image analysis software (version 4.1.0.0, Media Cybernetics, Rockville, MD, USA) was used to measure the size of the wounds by analyzing the images taken at each assessment point (day 0, 4, 8, 12, 16, 20 and 24). To evaluate the changes in the wounds over time, three different parameters were determined from the images as described earlier [27]: the (1) open wound area, (2) wound contraction area and (3) re-epithelization area (Sup. Figure 1). Calculations of the three parameters are shown below. 
(1). Open wound area:

$$
\left(\frac{A_{0}-\left(A_{0}-A_{G T}\right)}{A_{0}}\right) \times 100
$$

(2) Wound contraction:

$$
\left(\frac{A_{0}-\left(R_{G T}+A_{G T}\right)}{A_{0}}\right) \times 100
$$

(3) Re-epithelialization:

$$
\left(\frac{C_{G T}-A_{G T}}{A_{0}}\right) \times 100
$$

$A_{0}=$ Wound area at Day 0

$A_{G T}=$ Wound area at given time

$R_{G T}=$ Re-epithelialization at given time

$C_{G T}=$ Wound contraction at given time

\subsection{Statistical analysis}

A two tailed t-test was used to determine the difference between the swelling index $I_{\mathrm{s}}(\%)$ of nanofibers. A p values of $<0.05$ was considered the significant difference between the formulations.

The two- sample non-parametric statistical Mann-Whitney U-test was used to determine statistically significant differences in wound healing between groups in the animal study. Significant difference between the groups was set to $p<0.05$.

\section{Results and discussion}

The ideal wound dressing should, in general, be capable of maintaining optimal humidity at the wound site while removing excess exudate, be particle-free, non-toxic and non-allergenic, impermeable to bacteria, comfortable and conformable, require reduced dressing changes, be cost effective and have long shelf life $[3,8,29]$. However, an optimal treatment should be 
chosen based on the wound conditions, location and considering the specific patient's needs [16]. Although $\beta \mathrm{G}$ is commercially available in the form of a hydrogel, we aimed to develop alternative $\beta \mathrm{G}$-loaded wound dressing which would overcome the hydrogel-related limitations, i.e. limited use in treatment of exuding wounds.

Electrospinning is a versatile manufacturing method that forms nanofibrous dressings characterized by high surface area to volume ratio. Electrospinning opens new possibilities, as nanofibers can be used to treat various types of wounds and are comparable or superior to other modern wound dressings, such as hydrocolloids, hydrogels, and similar $[13,30]$. In addition, nanofiber mats are able to absorb wound exudate and provide a moist environment within the wound area. Moreover, the method is also suitable for forming nanofibers with active ingredients that might accelerate wound healing [13].

Although the ability of yeast $\beta \mathrm{G}$ to promote wound healing was first reported by Leibovich and Dannon already in 1980 [31], and $\beta G$ have been used in cosmetics and health promoting products for decades [32], nanofiberous wound dressings containing $\beta \mathrm{G}$ is still not available on the market. Thus, we were encouraged to develop electrospun nanofibers containing $\beta G$ as the active ingredient. Based on the preliminary results obtained with the needle electrospinning setup (results not shown), PEO and HPMC were selected as copolymers for the $\beta \mathrm{G}$-nanofibers, and the only polymers in the NoßG-nanofibers. To further optimize the formulation and to address potential upscaling of the production applying the Nanospider ${ }^{\mathrm{TM}}$ technology, we first optimized the spinning solutions.

\subsection{Optimization of electrospinning and resulting nanofibers}

\subsubsection{The polymer solutions and viscosity characteristics}

The properties of the polymer solution are one of the main factors affecting the transformation of the polymer solution into nanofibers by electrospinning [33]. All polymers applied in the formulation, namely HPMC; PEO and $\beta \mathrm{G}$, are water soluble. HPMC is an highly appreciated amorphous-state stabilizing carrier polymer in nanofibers [34]. PEO is known to greatly improve the spinability of HPMC even at small concentrations $(<0.1 \%)$ [35], and is an easily electrospinnable polymer, widely applied as a co-spinning agent [36]. Although the preliminary studies showed that $\beta \mathrm{G}$, in combination with HPMC and PEO, could be successfully electrospun from an aqueous solution, applying EtOH as a (co-)solvent made the 
polymer solutions less viscous and reduced the surface tension as compared to the pure aqueous solution (results not shown). Since the EtOH/water solvent system is environmentally acceptable, affordable and acceptable from the industrial point of view, this solvent system was preferred for the polymer solutions applied in the Nanospider ${ }^{\mathrm{TM}}$. Higher EtOH concentrations enabled the use of higher polymer concentrations in the $\beta \mathrm{G}-\mathrm{Sol}$ and NoßG-Sol, as compared to the concentrations in the Ref-Sols (Table 1); the polymer concentrations were higher than reported by Frenot et al. [23] who applied a 1:1 EtOH/water (v/v) solvent system for spinning HPMC in the needle electrospinning setup. Thus, a polymer concentration higher than $3 \%$ was applied in both $\beta \mathrm{G}-\mathrm{Sol}$ and NoßG-Sol. The HPMC2 (RefSol2) was more viscous as compared to HPMC1 (Ref-Sol1), therefore the upper limit of recommendable viscosity regarding the spinability was exceeded when Ref-Sol2 was tested in the Nanospider ${ }^{\mathrm{TM}}$. The lack of nanofiber formation is usually attributed to too viscous polymer solution used in spinning; too viscous solutions limit the formation of jets [37]. Thus, the Ref-Sol 3 was prepared with a 25\% reduction in HPMC2 concentration as compared to Ref-Sol2, and had a final HPMC content of $2.14 \%$. Different degree of substitution is known to affect the HPMC features [38], and explains the different viscosities of two HPMCderivatives tested. As we aimed to reach higher polymer concentrations, the HMPC1 was chosen for the $\beta \mathrm{G}-\mathrm{Sol}$ and No $\beta \mathrm{G}-\mathrm{Sol}$.

The lower viscosity of No $\beta \mathrm{G}-\mathrm{Sol}$ as compared to $\beta \mathrm{G}$-Sol (Figure 2 ), does not only relate to the absence of $\beta \mathrm{G}$ in the No $\beta \mathrm{G}-\mathrm{Sol}$, but also to the lower volume of water, since the hydrophilic polymers swell less in EtOH compared to water. It is known that the length of the polymers greatly influences the viscosity of the spinning solution, therefore the polymers with smaller molecule weights could potentially have been used to gain higher polymer concentrations [39]. However, an increase in polymer length induces polymer entanglement and prevents the formation of beads on the nanofibers and helps the formation of continuous nanofibers [37]. Thus, HPMC with lower molecule masses were not pursued. Spinning of both Ref-Sol1 and Ref-Sol3 resulted in nanofibers, however Ref-Sol1 formed only a very thin nanofiber layer on the substrate. Moreover, the layer was harder to peel off from the substrate than the other formulations. 


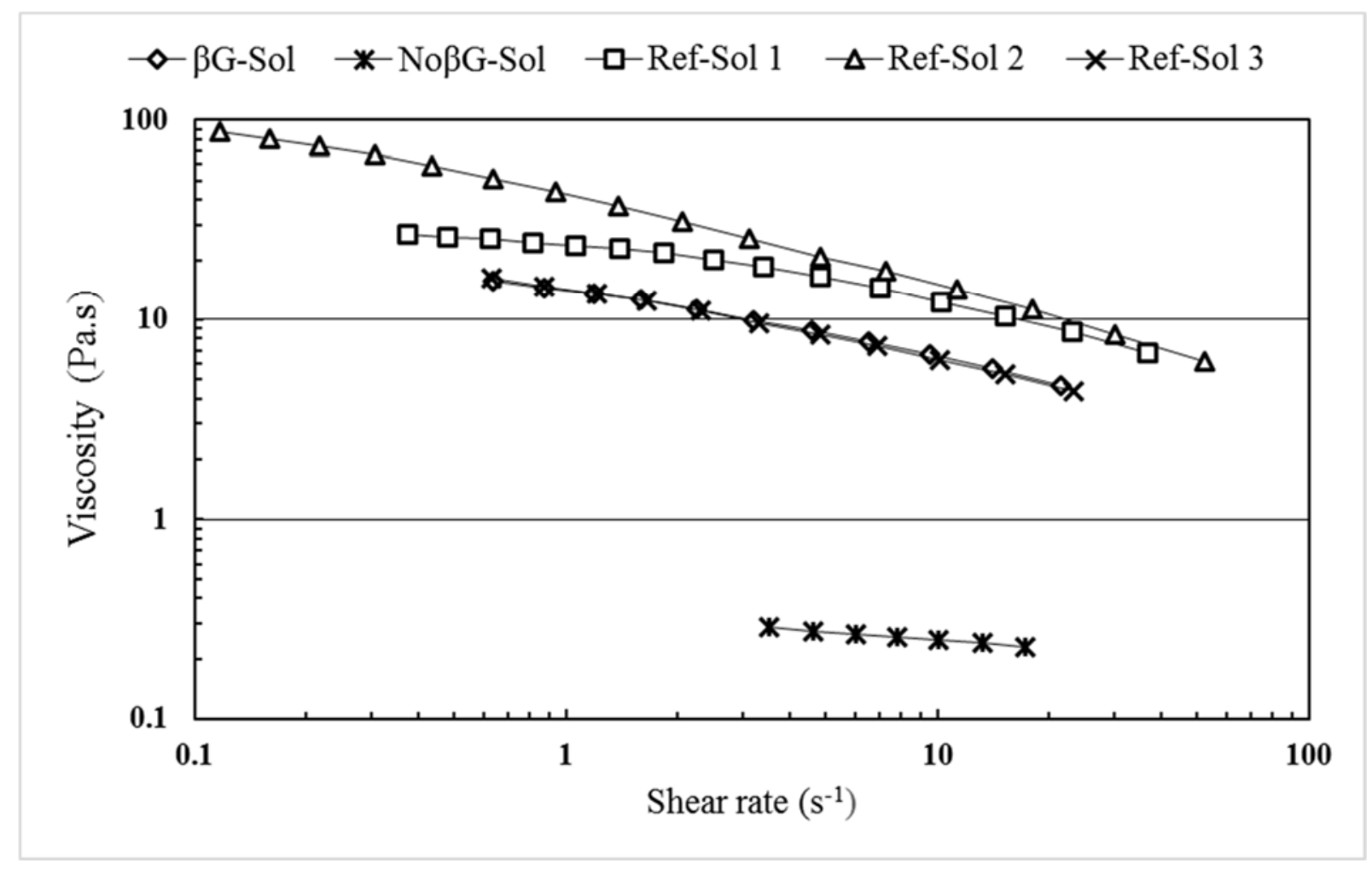

Figure 2. Flow viscosity of tested spinning solutions.

\subsubsection{Nanofiber Production by Needle-free Electrospinning}

To successfully produce electrospun nanofibers, not only the choice of polymer solutions, but also the processing parameters have to be optimal and controlled [40]. Foremost, it is important to consider the characteristics of the equipment that will be used in manufacturing [41]. Electrospinning has the advantage of producing the fibers in the nano-range, however comparing to other fiber producing methods, such as melt-blown, the production speed is rather slow [42]. By using needle-free electrospinning it is possible to produce higher volume of nanofiber sheets than with a traditional needle electrospinning setup $[41,43,44]$. The advantage of needle-free electrospinning is that there are no needles, nozzles or spinnerets that can be clogged, making the Nanospider ${ }^{\mathrm{TM}}$ technology a good candidate for industrial upscaling and the chosen technology for this study. Using the Elmarco Nanospider ${ }^{\mathrm{TM}}$ Lab, a pilot scale machine, the following parameters were successfully applied: the highest current of $80 \mathrm{kV}$, a spinning distance of $20 \mathrm{~cm}$ and the maximum carriage movement speed of 300 $\mathrm{mm} / \mathrm{s}$. To obtain different thicknesses of the final nanofiber webs, the speed of the substrate was varied between 2 and $4 \mathrm{~mm} / \mathrm{min}$. When applying the Nanospider ${ }^{\mathrm{TM}}$ Lab machine, it is important to coat the spinning wire sufficiently to continuously produce Taylor cones (liquid 
meniscuses from where the charged fluid jets are ejected) all along the wire. When the nanofiber mats were prepared using the substrate speed of $2 \mathrm{~mm} / \mathrm{min}$, the feed rate limitation was related to the higher viscosity of Ref-Sol1, and the spinning wire did not receive the needed amount of spinning solution to produce a steady stream of Taylor cones. With a pump modification on the Nanospider ${ }^{\mathrm{TM}}$ Lab feed system, it could be possible to feed the spinning wire more efficiently to increase the output, since the solutions exhibit shear thinning (Figure 2). $\beta \mathrm{G}-$ Sol performed similarly to Ref-Sol3, producing a steady stream of Taylor cones along the spinning wire of the Nanospider ${ }^{\mathrm{TM}} \mathrm{Lab}$. The viscosity of the $\beta \mathrm{G}$-Sol was close to the upper limit of what the Nanospider ${ }^{\mathrm{TM}}$ Lab can electrospin, and the solution contained the maximal dry content that the machine can handle within the current setup. The dry content in the NoßG-Sol could have been increased, without exceeding the viscosity limitation of the solution. However, since the dry content was in the same magnitude, the production speed was sufficient for both fibers, and the $40 \mathrm{~mL}$ batch volume was prepared within 30 minutes, and found to be suitable. Since the relative humidity affects the properties of the formed nanofibers to a high extent, and the higher humidity decreases the fiber diameter [45], the humidity and the temperature were monitored and controlled to be kept at $35 \pm 2 \%$ and $26 \pm 1$ ${ }^{\circ} \mathrm{C}$, respectively.

\subsubsection{Fourier-Transform Infrared Spectroscopy (FTIR) analysis}

FTIR spectra for the dried raw materials and the final electrospun nanofibers ( $\beta G$ and No $\beta G$ ) were recorded to confirm the presence of the respective polymers in the fibrous network comprising PEO (13.3\%, w/w) and HPMC (86.7\%, w/w) in the NoßG-nanofibers, and PEO $(10.0 \%, \mathrm{w} / \mathrm{w}), \mathrm{HPMC}(65.4 \%, \mathrm{w} / \mathrm{w})$ and $\beta \mathrm{G}(24.6 \%, \mathrm{w} / \mathrm{w})$ in the $\beta \mathrm{G}-$ nanofibers, respectively. Although distinctive band positions might allow the identification of polysaccharide structures and their composition [46], the interactions between the polymers, resulting in shifts in the band positions, as well as the overlapping bands originating from the different polymers in the sample, limit the interpretation of the spectra in terms of identifying the small spectral shifts of individual components in the mixture. However, the FTIR spectra obtained from the electrospun nanofibers demonstrated spectral features similar to the spectra obtained from the respective ingredients (Figure 3), although changes in the relative intensities and peak positions were observed. Bands characteristic for $\beta \mathrm{G}$ and HPMC in the region between 3600 and $3000 \mathrm{~cm}^{-1}$, assigned to the $-\mathrm{OH}$ stretching from intermolecular- and intramolecular hydrogen bonds, were observed for all $\beta \mathrm{G}$ - and HPMC containing materials, whereas the $\mathrm{CH}-$ 
stretching band in the area between 3000 and $2200 \mathrm{~cm}^{-1}$, was observed in all spectra. The $\beta G$ and HPMC exhibited, due to similar chemical structure, a more overlapping spectra than the PEO polymer. The $\mathrm{CH}_{2}$ assigned peaks at 842, 960, 1240 and $1278 \mathrm{~cm}^{-1}$ from PEO were more pronounced in spectra of the No $\beta G$ fibers than the $\beta G$ fiber spectra. The bands detected at around $1100 \mathrm{~cm}^{-1}$, that are formed through the combination of the ether and methylene group stretching vibrations in PEO and indicated as C-O-C [47], were also more visible in the NoßG than $\beta G$ fiber spectra. Thus, the intensity of these bands correlated to the PEO concentration in the nanofiber formulation.

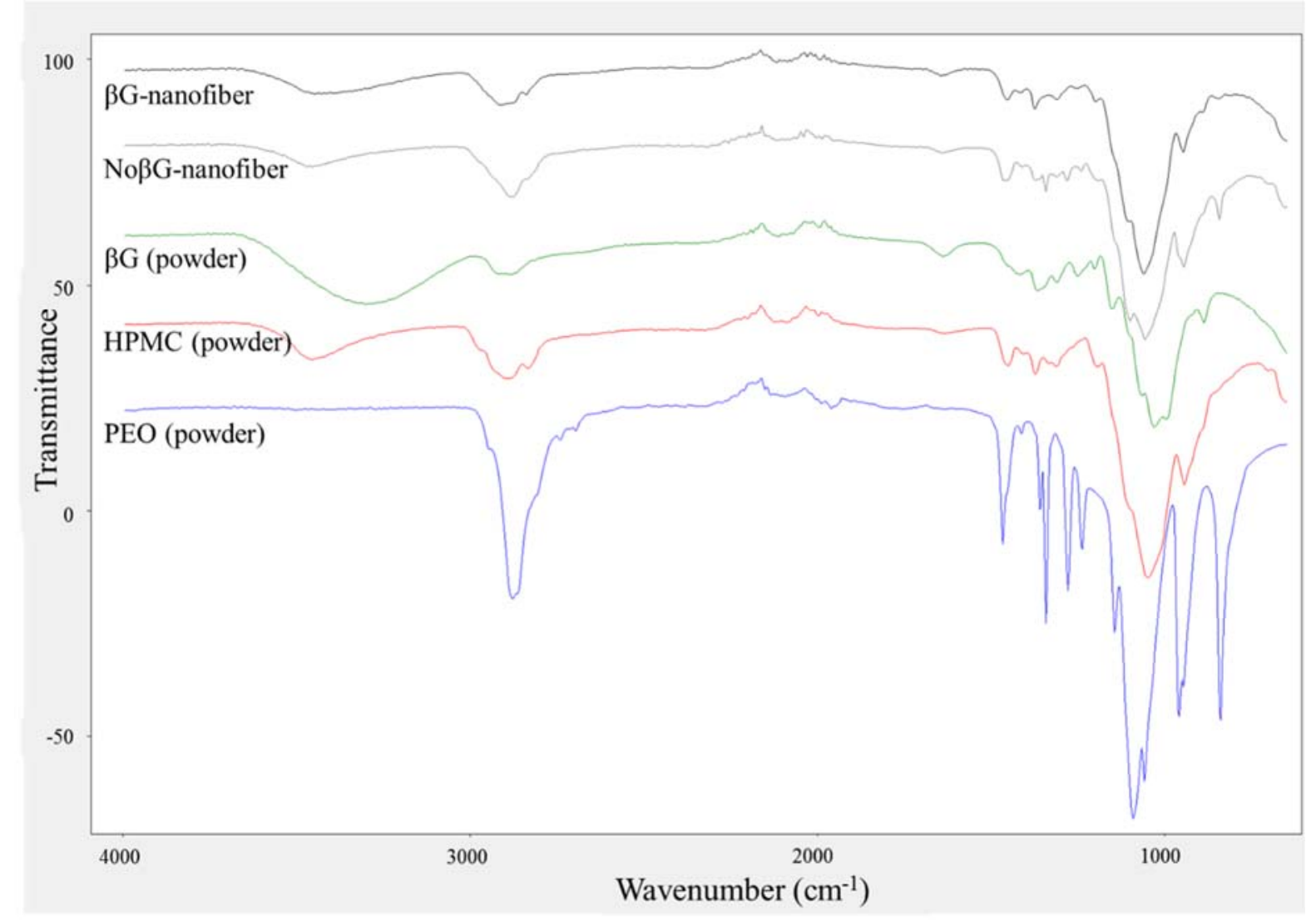

Figure 3. FTIR spectra of electrospun nanofibers ( $\beta \mathrm{G}$-and No $\beta \mathrm{G})$ and the corresponding polymers $\beta \mathrm{G}, \mathrm{HPMC}$ and $\mathrm{PEO}$.

\subsubsection{Morphology of nanofibers}

The morphology and dimensions of electrospun nanofibers provide a good indication of the quality of electrospinning, including the choice of polymers, their concentrations, choice of solvents, the machine and applied settings, as well as environmental settings (temperature and humidity). 
The FE-SEM pictures of the electrospun nanofibers confirmed the fiber formation. The diameter of the electrospun nanofibers was $110 \pm 74 \mathrm{~nm}$ and $180 \pm 95 \mathrm{~nm}$ for nanofibers made from $\beta \mathrm{G}$-Sol (Figure 4A) and No $\beta$ G-Sol (Figure 4B), and $100 \pm 50 \mathrm{~nm}$ and $81 \pm 39 \mathrm{~nm}$ for nanofibers made from Ref-Sol 1 (Figure 4C) and Ref-Sol 3 (Figure 4D), respectively. The increased diameter observed with increasing polymer concentrations (given in Table 1), is in accordance with reports from previous studies [40,45]. All nanofibers displayed the same structural forms, namely the ribbon shaped fibers. The formation of ribbon shaped/branched fibers might be due to the rapid evaporation of solvent from the outer part of the jet, and the subsequent diffusion of solvent from the core out through the surface of the fiber on the collector [48]. Changing the relative humidity could prevent the formation of ribbon shaped nanofibers and stabilize the jet [45]. However, the production of less branched fibers does not necessarily support a more effective cell mobility and three-dimensional cell migration in wound healing [49], especially considering the rapid gelling of the nanofibers in this study (results given in Section 3.1.5). In addition, the relatively low humidity applied in this study $(35 \pm 2 \%)$ has been shown to lead to ticker nanofibers with a more homogeneous size distribution, and to decrease the beads formation, that might be a problem under the conditions of higher humidity and polymer concentrations, as well as with a long distance to the collector [45]. 

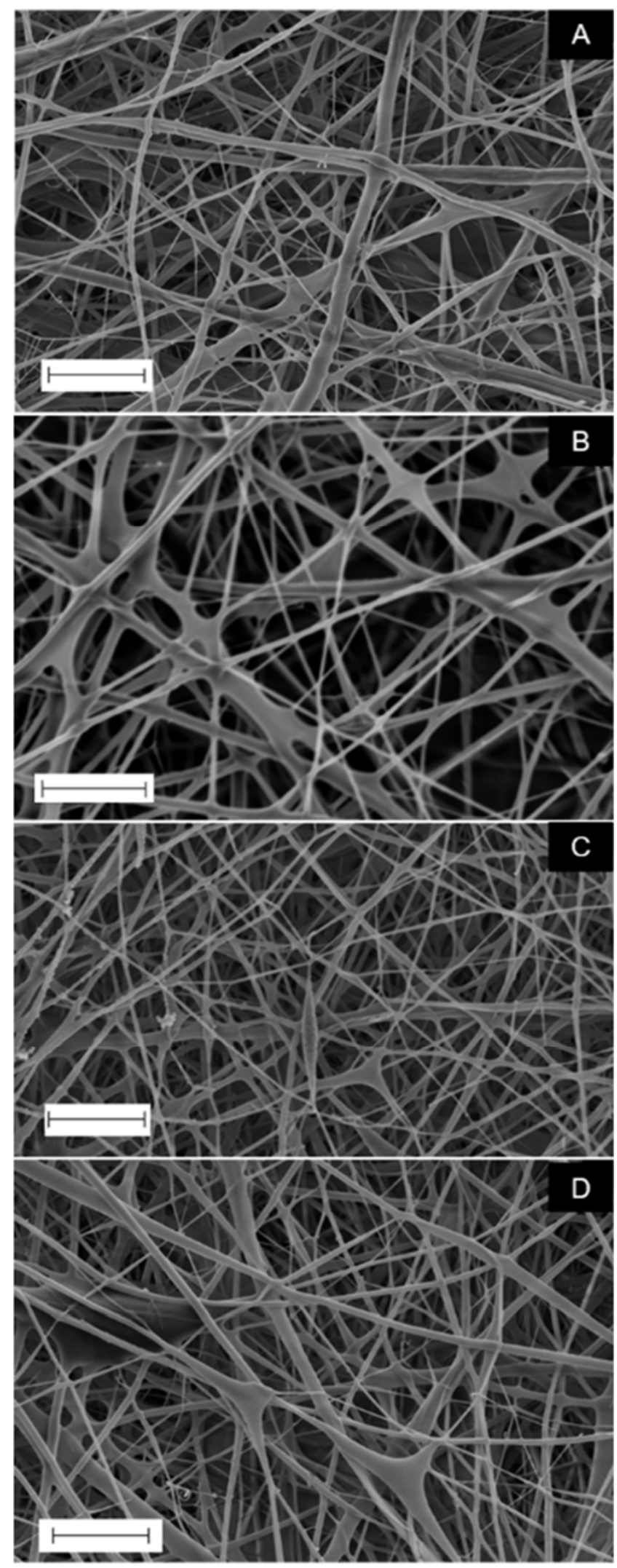

Figure 4. SEM pictures of electrospun nanofibers made from A: $\beta$ G-Sol. B: No $\beta$ G-Sol. C: Ref-Sol1. D: Ref-Sol3 polymer spinning solutions, respectively. All scale bars are $1 \mu \mathrm{m}$. 


\subsubsection{The nanofiber swelling capacity}

The dry nanofiber dressing is supposed to be applied directly onto the high to moderately exudation wound, and to absorb and retain the exudate onto the wound by forming an in situ gel (Figure 1) that allows autolytic debridement, while promoting a successful wound healing environment. The capability of swelling is therefore crucial for the optimal therapy and was thus investigated.

The $\beta$ G-nanofiber had a swelling index $\left(\mathrm{I}_{\mathrm{s}}\right)$ of $1287 \pm 109$ and the No $\beta \mathrm{G}$-nanofiber of $1537 \pm$ $141 \%(\mathrm{w} / \mathrm{w})$, respectively. Similar level of swelling capacity was reported for alginate dressing, that can absorb up to 20 times their own weight in fluid [29]. The average weight of the $\beta$ G-nanofibers and NoßG-nanofibers before swelling was $134 \mathrm{mg}$ and $190 \mathrm{mg}$, respectively. There was no significant differences in the swelling index for the two fiber formulations. Although both fibers hydrated very rapidly, the $\beta \mathrm{G}$-nanofiber seemed to hydrate somewhat slower than the No $\beta G$-nanofiber. During the swelling, the $\beta G$-nanofiber retained air trapped under the fiber (Sup. Figure 1B), whereas the NoßG-nanofiber (Sup. Figure 1C) retained air within the gel. This might indicate that the $\beta G$-nanofiber would have retained more liquid if allowed to soak in the aqueous media for a longer time. However, both formulations were allowed to soak for only 60 seconds to avoid obtaining too liquefied fibers that would be impossible to remove from the Petri dish, and to allow excess aqueous media to be removed in a reproducible way. Thus, the relatively high swelling capacity clearly makes the nanofibers less mechanically stable. For comparison, the Curdlan/PVA nanofibers obtained for nanofiber scaffolds made for tissue engineering [50], reached a swelling saturation of approximately $170 \%$ after 24 hours swelling in PBS at $37{ }^{\circ} \mathrm{C}$.

The lower mechanical strength and fast swelling of our fibers can be contributed to the use of biodegradable and bioabsorbable polymers selected. The high swelling capacity and rather poor mechanical strength of this novel dressing indicate that it does not have to be removed from the wound; something that is highly advantageous considering patient comfort and pain associated to dressing exchange [2]. Moreover, since it is a transparent dressing, the wound healing progression and tissue can be observed although the wound is covered with the dressing. 


\subsection{Cytotoxicity of electrospun nanofibers}

The toxicity of the nanofibers was tested in vitro using MTT assay method. Three different concentrations of the $\beta$ G-nanofibers, No $\beta G$-nanofibers and $\beta \mathrm{G} 2.5 \%(1,10,100 \mu \mathrm{g} / \mathrm{mL})$ were applied on the keratinocytes. Neither the $\beta G$-nanofibers nor the No $\beta G$-nanofibers and $\beta G$ $2.5 \%$, exhibited any toxicity up to the concentration of $100 \mu \mathrm{g} / \mathrm{mL}$, which is in agreement with Choromanska et al. [51]. These results suggest that nanofibers will not affect the cell viability during the proliferation phase of wound healing.

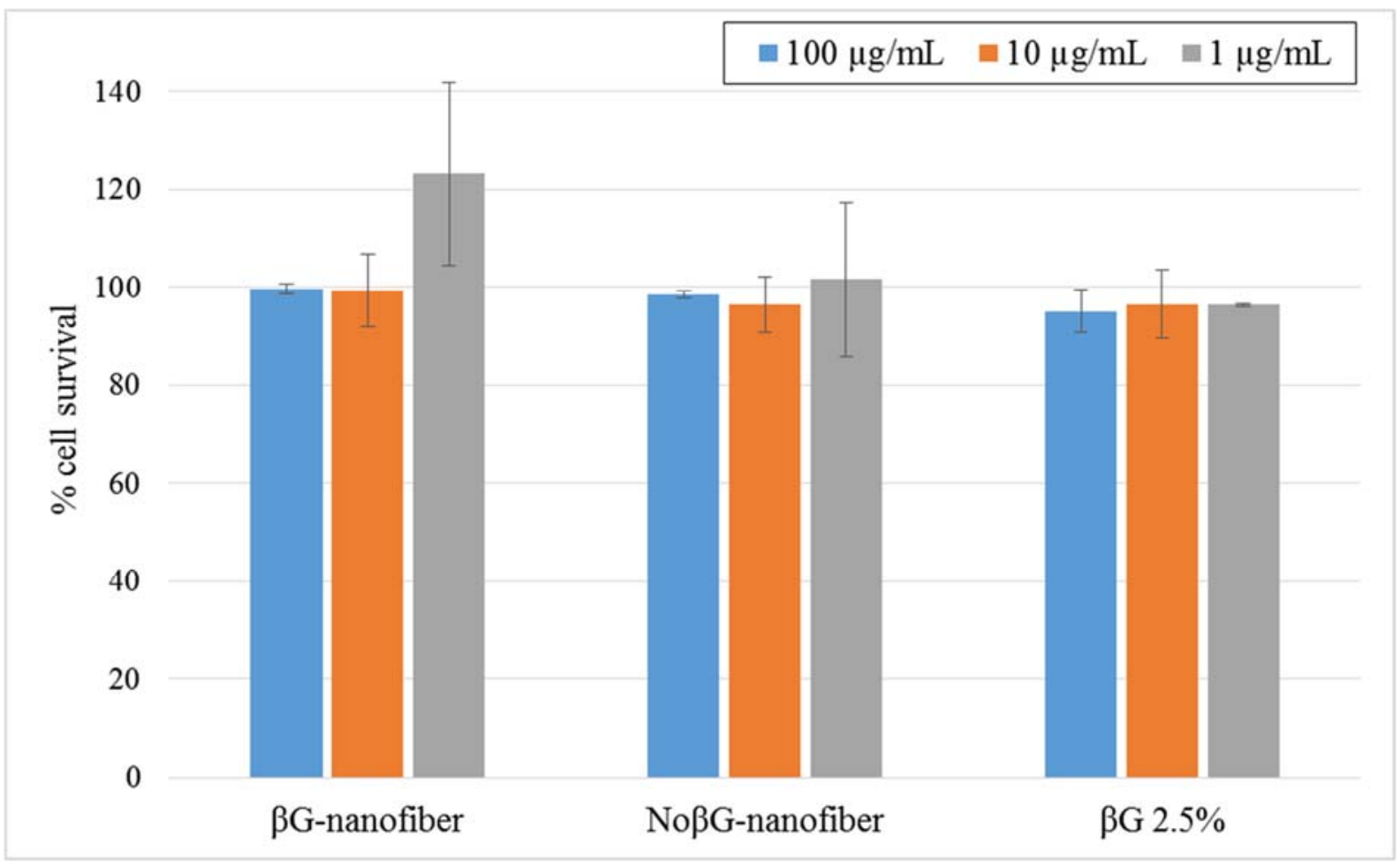

Figure 5. HaCaT cells toxicity as assessed by the MTT-assay. Three different concentrations $(1,10$, and $100 \mu \mathrm{g} /)$ of solubilized nanofibers or active ingredient $(\beta \mathrm{G})$ were applied on keratinocytes. Results are given as mean of two independent experiments.

\subsection{In vivo wound healing in diabetic mice}

In the final stage of this study, the wound healing potential of the novel nanofibers, both with $\beta \mathrm{G}$ ( $\beta \mathrm{G}$-nanofibers) and without $\beta \mathrm{G}$ (NoßG-nanofibers) was tested in vivo. Three different doses of $\beta G$-nanofibers containing 190, 370 and $990 \mu \mathrm{g}$ of $\beta \mathrm{G}$ (Table 1), respectively, and the NoßG-nanofiber were administered onto the excisional wounds in the diabetic $\mathrm{db} / \mathrm{db}$ mice.

Diabetic $\mathrm{db} / \mathrm{db}$ wound healing model is known to provide a well-defined wound bed that heals both from contraction and re-epithelialization, and is considered a reproducible and valuable model for testing experimental wound healing treatments [52]. During the treatment, no 
adverse reactions were observed in any animal group. Only one animal in the Low $\beta G$ group was removed due to subcutaneous abscess and rapid weight loss. However, these changes did not appear to be directly related to the treatment. All treatments were applied under occlusive cover, to retain the formulations in place, prevent moisture loss, and the animals from removing the treatment. The use of occlusive dressing is the current state-of-the-art as it is used in clinical wound care to prevent moisture loss and accelerate healing in the acute, chronic and infected wounds [29]. The use of occlusive dressing assists wound healing [53]. Although the occlusion in itself improve wound healing, the animals in the negative control group (water treatments) exhibited an increase in wounded area on day 4 (103.1\% of initial wound), however, on day 8 the wound contraction was detected (74.2\% of initial wound); the contraction was in the same magnitude as observed for the NoßG group (73.5\% of initial wound area). After day 8, all treatments were significantly better than the negative control and provided improved healing conditions $(\mathrm{p}<0.05)$. These results indicate that the beneficial effect of $\beta \mathrm{G}$-nanofibers on wound healing can not only be attributed to the active ingredient $\beta \mathrm{G}$, but that the nanofibers as wound dressing also improve wound healing. The HPMC and PEO applied as copolymers in the nanofibers seem to contribute in providing an optimal moist environment for wound healing. The wound healing mechanisms of $\beta G$ is derived from the activation of the macrophages and the subsequent signaling [14,54], however, the results indicate that this healing potential is enhanced by the nanofibers.

All three groups treated with $\beta$ G-nanofibers exhibited significantly improved wound closure $(p<0.05)$ as compared to the NoßG group. The results shown in Figure 6 and 7 also demonstrate that the wound closure started faster during the $\beta G$-nanofiber treatment (76.8$82.3 \%)$ than the positive control $(97.9 \%)$ on day 4 ; however on day 8 the wound closure in $\beta G$ treated groups was similar to the wound closure in mice treated with positive control. Unfortunately, we could not confirm the dose-response for the $\beta G$-nanofibers, most probably because the effective dose was reached already with the lowest dose applied. Thus, it would be interesting to investigate the wound healing at lower $\beta \mathrm{G}$ dosages. This could be achieved either by lowering the concentration of $\beta \mathrm{G}$ in the electrospun nanofibers, the amount of nanofibers, or by reducing the frequency of dosing/application onto the wound. Lower doses or reduced frequency of treatment would have both economic benefits and be advantageous for the patient compliance and effect of the treatment. The less frequent treatment applied in the nanofiber groups might also have caused less stress to the mice and positively attribute to 
the healing process. It is known that the stress can inhibit wound healing in animals trough affecting the functional wound healing pathways [55].

The wound healing potential of tested treatments was reproducibly demonstrated through the small standard deviations from the mean value of the assessed wound closure, contraction and re-epithelization (Figure 6). All treated groups had a standard error mean $(\mathrm{SEM})<3.7$, with an exception of the NoßG group (SEM of 8.9) in the wound closure assessment. The NoßG ( $\beta \mathrm{G}$-free nanofibers) are not expected to improve wound healing to a greater extent. In our previous study [27] we confirmed the healing potential of $\beta G$ in wound healing, and present results additionally confirm the positive effects of $\beta G$ in formulation. The improved wound healing in the $\beta G$-nanofiber treated groups as compared to the NoßG-nanofiber group seem to be attributed to the increased wound contraction rather than to an increase in the reepithelization (Figure 7). The wound healing contraction, attributed to the panniculus carnosus muscle, is known to be more pronounced in murine than in human [52]. Due to the rapid gelling of the components within the nanofiber dressing, it is likely that the effects are not due to mechanistic contraction, but rater elicited by a cellular response to $\beta \mathrm{G}$. When accounting for the re-epithelization in the diabetic $\mathrm{db} / \mathrm{db}$ mice model, the contraction of the skin will mask some of re-epithelized tissue due to compaction of the wound [56], leading to a decline in the cumulative granulation. This decline in re-epithelization can be seen in all groups with exception of the water-treated (negative control) group (Figure 6).

In our previous study on wound healing potential of $\beta G$ formulated as hydrogel, we failed to prove its superiority as wound dressing. We suggested that the failure can be due to an adverse effect of Carbopol 971P NF in the hydrogel formulation [27]. The current in vivo results support the suggested explanation. In the present study, the excipients applied in the nanofibers seem not to interfere with the healing potential of $\beta G$, but rather to have a positive effect. In addition, nanofibers as dressing improved wound healing physically. There are several publications reporting the use of nanofibers for wound treatment in mice $[11,57,58]$ and rats [59-62]; however, to the best of our knowledge this is the first work on in vivo wound healing of a in situ gel forming $\beta \mathrm{G}$-nanofibers, with $\beta \mathrm{G}$ isolated from beakers yeast. There is only one publication on electrospun poly(lactic-co-glycolic acid) (PLGA) nanofibers loaded with $\beta \mathrm{G}$ from Aureobasidium pullulans [63]. Kim and co-authors observed small differences between the treatment with $\beta$ G-containing nanofibers and PLGA alone. However, they found that $\beta G$ stimulated the cells that helped with re-epithelialization, proliferation and angiogenesis. The use of PLGA as a carrier provides a more rigid nanofiber structure that is 
less prone to lose its integrity, since PLGA degrades by hydrolysis and does not gel, contrary to PEO and HPMC used in our study. Considering that the structure of $\beta G$ is crucial for the cellular response, and plays a significant role in the bioactivity of $\beta G$, these two studies are not directly comparable. In addition, the evidence of the wound healing potential of $\beta \mathrm{G}$ from Aureobasidium pullulans is rather limited $[32,64]$.

The novel $\beta G$-containing nanofibers bear the potential to serve as an advanced wound dressing for exuding wounds. The manufacturing scale up should not present serious challenge within industrial manufacturing.

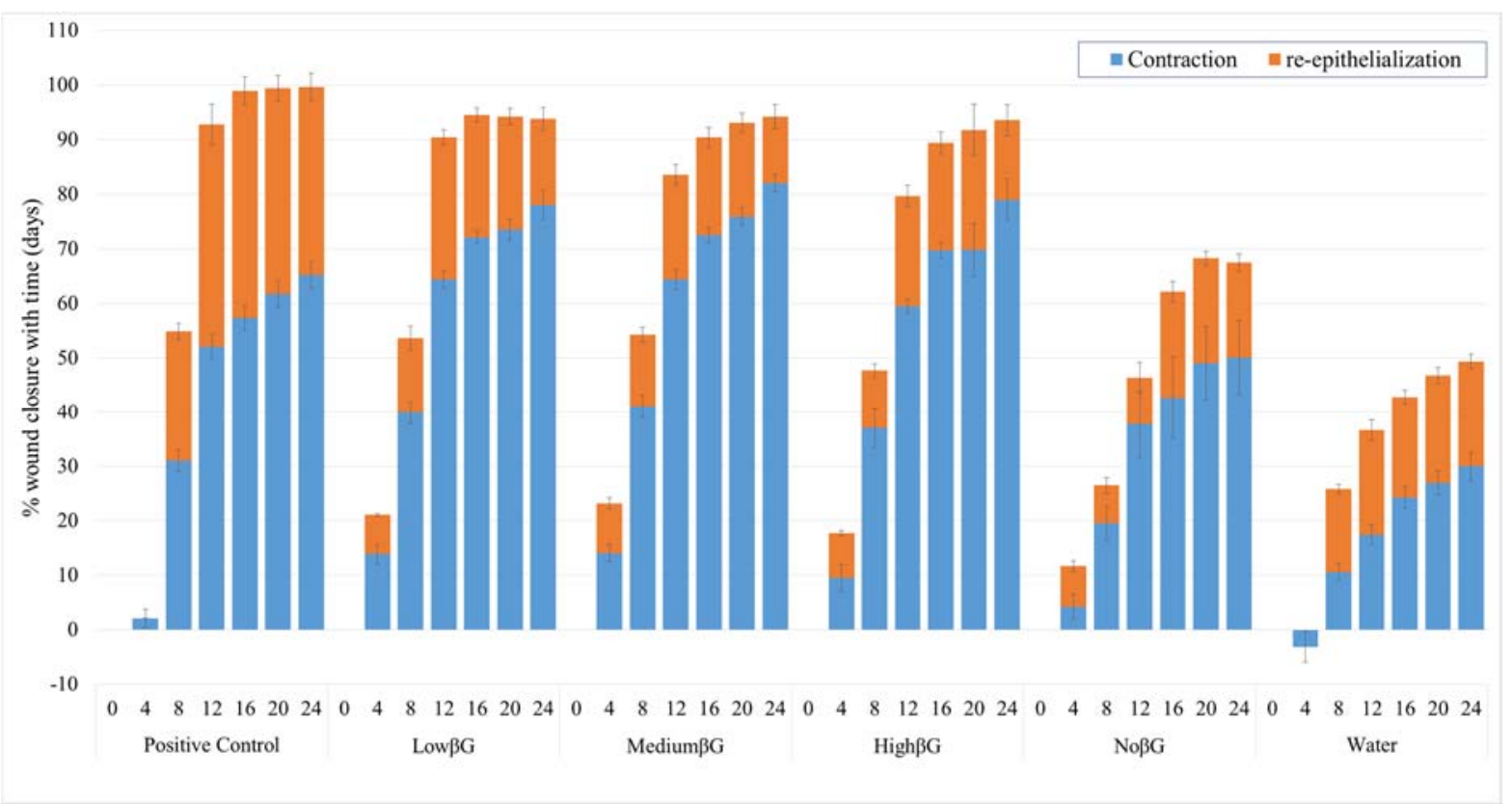

Figure 6. The relative contribution from contraction and re-epithelialization on the total wound closure in the different treatment groups $(\% \pm \operatorname{SEM})(n=10)$. 


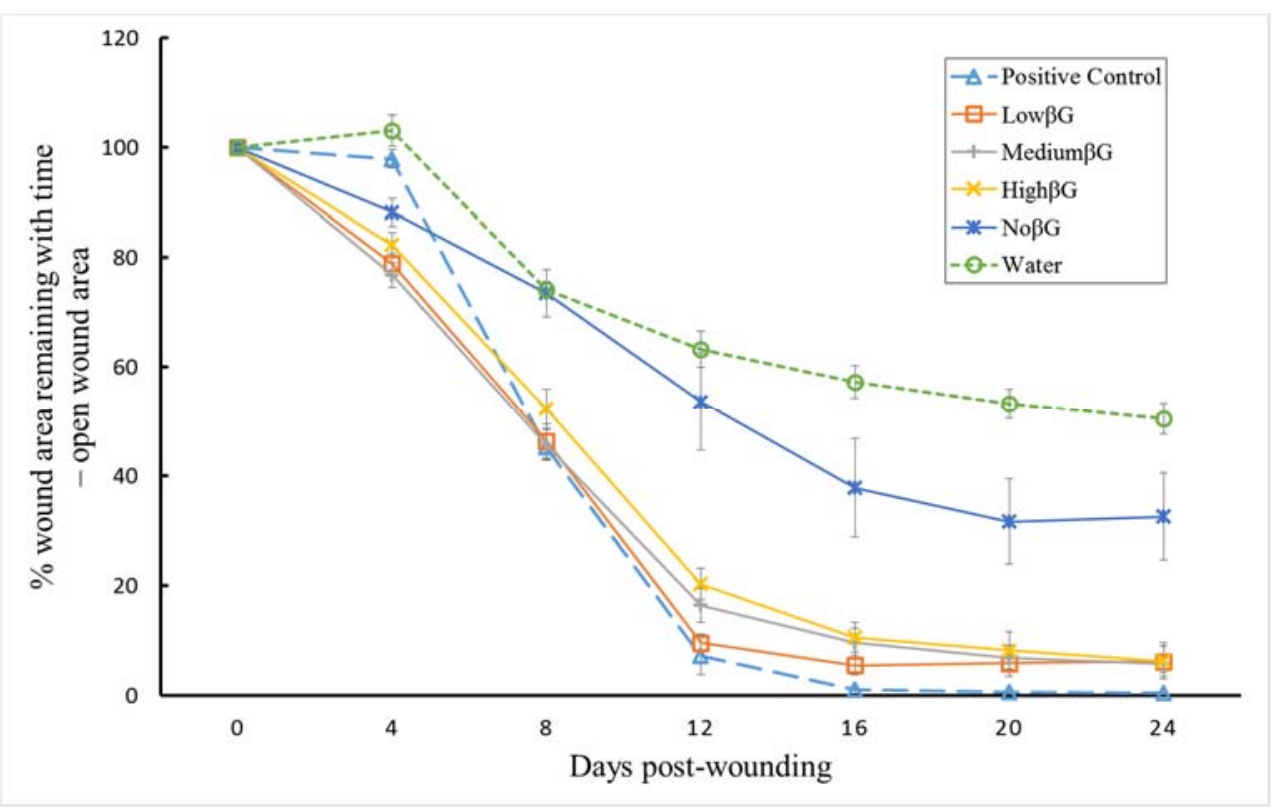

Figure 7. The relative wound closure area after different treatments $( \pm \operatorname{SEM})(n=10)$

\section{Conclusions}

The Nanospider ${ }^{\mathrm{TM}}$ technology applied in the production of electrospun $\beta$ G-nanofibers was proven to be a reproducible and reliable method to manufacture nanofibers of desired features considering the treatment of medium exuding wounds. Due to the high capacity of water retention and rapid swelling, the nanofibers formed a hydrogel in situ upon administration onto the wound. The novel formulation exhibited no cellular toxicity in the in vitro MTT assay. The wound healing assessment in the wound model of hard-to-heal wounds confirmed that the $\beta G$-nanofiber significantly improved the healing as compared to the NoßGnanofibers. The findings can be applied as a ground for further testing in human.

\section{Acknowledgement}

The authors would like to thank The Research Council Norway for funding (grant number 240123/O30) the PhD position of Jostein Grip. We would like to acknowledge Cica Biomedical Ltd (North Yorkshire, England) and Dr. Jeff Hart, Managing Director and Dr. Andrea Bell, Principal Scientist, for support with the in vivo work. We would also like to acknowledge Ivo Laidmäe and Jyrki T. Heinämäki for introducing us to electrospinning at the Department of Pharmacy, University of Tartu. 


\section{Declaration of interest}

Jostein Grip is affiliated with Biotec BetaGlucan AS, as a part of an industry sponsored PhDprogram.

\section{References}

[1] G. Han, R. Ceilley, Chronic wound healing: a review of current management and treatments, Adv. Ther. 34 (2017) 599-610. doi:10.1007/s12325-017-0478-y.

[2] K. Woo, G. Sibbald, K. Fogh, C. Glynn, D. Krasner, D. Leaper, J. Österbrink, P. Price, L. Teot, Assessment and management of persistent (chronic) and total wound pain, Int. Wound J. 5 (2008) 205-215. doi:10.1111/j.1742-481X.2008.00483.x.

[3] J.S. Boateng, K.H. Matthews, H.N.E. Stevens, G.M. Eccleston, Wound healing dressings and drug delivery systems: a review, J. Pharm. Sci. 97 (2008) 2892-2923. doi:10.1002/jps.21210.

[4] R.G. Frykberg, J. Banks, Challenges in the Treatment of Chronic Wounds, Adv. Wound Care. 4 (2015) 560-582. doi:10.1089/wound.2015.0635.

[5] H. Sorg, D.J. Tilkorn, S. Hager, J. Hauser, U. Mirastschijski, Skin wound healing: an update on the current knowledge and concepts, Eur. Surg. Res. 58 (2017) 81-94. doi:10.1159/000454919.

[6] S.L. Percival, S.M. McCarty, B. Lipsky, Biofilms and wounds: an overview of the evidence, Adv. Wound Care. 4 (2015) 373-381. doi:10.1089/wound.2014.0557.

[7] J.G. Powers, C. Higham, K. Broussard, T.J. Phillips, Wound healing and treating wounds Chronic wound care and management, J. Am. Acad. Dermatol. 74 (2016) 607625. doi:10.1016/j.jaad.2015.08.070.

[8] J. Boateng, O. Catanzano, Advanced therapeutic dressings for effective wound healing - a review, J. Pharm. Sci. 104 (2015) 3653-3680. doi:10.1002/jps.24610.

[9] J. Wang, M. Windbergs, Functional electrospun fibers for the treatment of human skin wounds, Eur. J. Pharm. Biopharm. 119 (2017) 283-299. doi:10.1016/j.ejpb.2017.07.001.

[10] J.S. Choi, H.S. Kim, H.S. Yoo, Electrospinning strategies of drug-incorporated nanofibrous mats for wound recovery, Drug Deliv. Transl. Res. 5 (2015) 137-145. doi:10.1007/s13346-013-0148-9.

[11] Q. Li, Y. Niu, H. Diao, L. Wang, X. Chen, Y. Wang, L. Dong, C. Wang, In situ sequestration of endogenous PDGF-BB with an ECM-mimetic sponge for accelerated wound healing, Biomaterials. 148 (2017) 54-68. doi:10.1016/j.biomaterials.2017.09.028.

[12] S. Thakkar, M. Misra, Electrospun polymeric nanofibers: New horizons in drug delivery, Eur. J. Pharm. Sci. 107 (2017) 148-167. doi:10.1016/j.ejps.2017.07.001. 
[13] M. Liu, X.-P. Duan, Y.-M. Li, D.-P. Yang, Y.-Z. Long, Electrospun nanofibers for wound healing, Mater. Sci. Eng. C. 76 (2017) 1413-1423. doi:10.1016/j.msec.2017.03.034.

[14] J. Ma, D.M. Underhill, $\beta$-glucan signaling connects phagocytosis to autophagy, Glycobiology. 23 (2013) 1047-1051. doi:10.1093/glycob/cwt046.

[15] C.-H. Lee, S.-H. Chang, W.-J. Chen, K.-C. Hung, Y.-H. Lin, S.-J. Liu, M.-J. Hsieh, J.H.S. Pang, J.-H. Juang, Augmentation of diabetic wound healing and enhancement of collagen content using nanofibrous glucophage-loaded collagen/PLGA scaffold membranes, J. Colloid Interface Sci. 439 (2015) 88-97. doi:10.1016/j.jcis.2014.10.028.

[16] B. King, S. Barrett, K.F. Cutting, Clinical evaluation of a bioactive beta-glucan gel in the treatment of "hard-to-heal" wounds, J. Wound Care. 26 (2017) 58-63. doi:10.12968/jowc.2017.26.2.58.

[17] K.F. Cutting, The cost-effectiveness of a novel soluble beta-glucan gel, J. Wound Care. 26 (2017) 228-234. doi:10.12968/jowc.2017.26.5.228.

[18] R. Engstad, T. Nøkland, Glucans, US9623043 B2, 2017. https://www.google.com/patents/US9623043.

[19] C.S. Engstad, R.E. Engstad, J.O. Olsen, B. Østerud, The effect of soluble $\beta$-1,3-glucan and lipopolysaccharide on cytokine production and coagulation activation in whole blood, Int. Immunopharmacol. 2 (2002) 1585-1597. doi:10.1016/S15675769(02)00134-0.

[20] R.E. Engstad, B. Robertsen, Specificity of a beta-glucan receptor on macrophages from Atlantic salmon (Salmo salar L.)., Dev. Comp. Immunol. 18 (1994) 397-408. doi:10.1016/0145-305X(94)90005-1.

[21] H. Zhang, L. Zhang, Q. Jia, C. Shi, J. Yang, Preparation of porous nylon 6 fiber via electrospinning, Polym. Eng. Sci. 55 (2015) 1133-1141. doi:10.1002/pen.23984.

[22] R. Stepanyan, A.V. Subbotin, L. Cuperus, P. Boonen, M. Dorschu, F. Oosterlinck, M.J.H. Bulters, Nanofiber diameter in electrospinning of polymer solutions: model and experiment, Polymer (Guildf). 97 (2016) 428-439. doi:10.1016/j.polymer.2016.05.045.

[23] A. Frenot, M.W. Henriksson, P. Walkenström, Electrospinning of cellulose-based nanofibers, J. Appl. Polym. Sci. 103 (2007) 1473-1482. doi:10.1002/app.24912.

[24] D.L. Ho, B. Hammouda, S.R. Kline, W.R. Chen, Unusual phase behavior in mixtures of poly(ethylene oxide) and ethyl alcohol, J. Polym. Sci. Part B Polym. Phys. 44 (2006) 557-564. doi:10.1002/polb.20726.

[25] J.S. Boateng, H. V. Pawar, J. Tetteh, Polyox and carrageenan based composite film dressing containing anti-microbial and anti-inflammatory drugs for effective wound healing, Int. J. Pharm. 441 (2013) 181-191. doi:10.1016/j.ijpharm.2012.11.045.

[26] Standard, EN 13726-1 Test methodes for primary wound dressings - Part 1: Aspects of absorbancy, Fluid Affin. Amorph. Hydrogel Dressings. (2002).

[27] J. Grip, R.E. Engstad, I. Skjæveland, N. Škalko-Basnet, A.M. Holsæter, Sprayable Carbopol hydrogel with soluble beta-1,3/1,6-glucan as an active ingredient for wound healing - development and in-vivo evaluation, Eur. J. Pharm. Sci. 107 (2017) 24-31. doi:10.1016/j.ejps.2017.06.029. 
[28] Home Office, Guidance on the operation of the Animals (Scientific Procedures) Act 1986, 2014. https://www.gov.uk/government/publications/operation-of-aspa.

[29] K. Vowden, P. Vowden, Wound dressings: principles and practice, Surg. 35 (2017) 489-494. doi:10.1016/j.mpsur.2017.06.005.

[30] P. Zahedi, I. Rezaeian, S.O. Ranaei-Siadat, S.H. Jafari, P. Supaphol, A review on wound dressings with an emphasis on electrospun nanofibrous polymeric bandages, Polym. Adv. Technol. 21 (2010) 77-95. doi:10.1002/pat.1625.

[31] S.J. Leibovich, D. Danon, Promotion of wound repair in mice by application of glucan., J. Reticuloendothel. Soc. 27 (1980) 1-11.

[32] B. Du, Z. Bian, B. Xu, Skin health promotion effects of natural beta-glucan derived from cereals and microorganisms: a review, Phyther. Res. 28 (2014) 159-166. doi:10.1002/ptr.4963.

[33] W.K. Son, J.H. Youk, T.S. Lee, W.H. Park, The effects of solution properties and polyelectrolyte on electrospinning of ultrafine poly(ethylene oxide) fibers, Polymer (Guildf). 45 (2004) 2959-2966. doi:10.1016/j.polymer.2004.03.006.

[34] U. Paaver, J. Heinämäki, I. Laidmäe, A. Lust, J. Kozlova, E. Sillaste, K. Kirsimäe, P. Veski, K. Kogermann, Electrospun nanofibers as a potential controlled-release solid dispersion system for poorly water-soluble drugs, Int. J. Pharm. 479 (2015) 252-260. doi:10.1016/j.ijpharm.2014.12.024.

[35] A. Balogh, B. Farkas, G. Verreck, J. Mensch, E. Borbás, B. Nagy, G. Marosi, Z.K. Nagy, AC and DC electrospinning of hydroxypropylmethylcellulose with polyethylene oxides as secondary polymer for improved drug dissolution, Int. J. Pharm. 505 (2016) 159-166. doi:10.1016/j.ijpharm.2016.03.024.

[36] M. Pakravan, M.C. Heuzey, A. Ajji, A fundamental study of chitosan/PEO electrospinning, Polymer (Guildf). 52 (2011) 4813-4824. doi:10.1016/j.polymer.2011.08.034.

[37] N. Bhardwaj, S.C. Kundu, Electrospinning: A fascinating fiber fabrication technique, Biotechnol. Adv. 28 (2010) 325-347. doi:10.1016/j.biotechadv.2010.01.004.

[38] N. Nyamweya, S.W. Hoag, Assessment of polymer-polymer interactions in blends of HPMC and film forming polymers by modulated temperature differential scanning calorimetry, Pharm. Res. 17 (2000) 625-631. doi:10.1023/A:1007585403781.

[39] P. Gupta, C. Elkins, T.E. Long, G.L. Wilkes, Electrospinning of linear homopolymers of poly(methyl methacrylate): exploring relationships between fiber formation, viscosity, molecular weight and concentration in a good solvent, Polymer (Guildf). 46 (2005) 4799-4810. doi:10.1016/j.polymer.2005.04.021.

[40] J. Pelipenko, P. Kocbek, J. Kristl, Critical attributes of nanofibers: Preparation, drug loading, and tissue regeneration, Int. J. Pharm. 484 (2015) 57-74. doi:10.1016/j.ijpharm.2015.02.043.

[41] R. Nayak, R. Padhye, I.L. Kyratzis, Y.B. Truong, L. Arnold, Recent advances in nanofibre fabrication techniques, Text. Res. J. 82 (2012) 129-147. doi:10.1177/0040517511424524.

[42] L. Persano, A. Camposeo, C. Tekmen, D. Pisignano, Industrial upscaling of 
electrospinning and applications of polymer nanofibers: a review, Macromol. Mater. Eng. 298 (2013) 504-520. doi:10.1002/mame.201200290.

[43] T. Grothe, C. Groaßerhode, T. Hauser, P. Kern, K. Stute, A. Ehrmann, Needleless electrospinning of PEO nanofiber mats, in: Proc. Second Int. Conf. Mech. Mater. Struct. Eng. (ICMMSE 2017), Atlantis Press, Paris, France, 2017: pp. 54-58. doi:10.2991/icmmse-17.2017.9.

[44] M.H. El-Newehy, S.S. Al-Deyab, E.-R. Kenawy, A. Abdel-Megeed, Nanospider Technology for the Production of Nylon-6 Nanofibers for Biomedical Applications, J. Nanomater. 2011 (2011) 1-8. doi:10.1155/2011/626589.

[45] J. Pelipenko, J. Kristl, B. Janković, S. Baumgartner, P. Kocbek, The impact of relative humidity during electrospinning on the morphology and mechanical properties of nanofibers, Int. J. Pharm. 456 (2013) 125-134. doi:10.1016/j.ijpharm.2013.07.078.

[46] M. Kačuráková, P. Capek, V. Sasinková, N. Wellner, A. Ebringerová, FT-IR study of plant cell wall model compounds: pectic polysaccharides and hemicelluloses, Carbohydr. Polym. 43 (2000) 195-203. doi:10.1016/S0144-8617(00)00151-X.

[47] I. Pucić, T. Jurkin, FTIR assessment of poly(ethylene oxide) irradiated in solid state, melt and aqeuous solution, Radiat. Phys. Chem. 81 (2012) 1426-1429. doi:10.1016/j.radphyschem.2011.12.005.

[48] A.J. Guenthner, S. Khombhongse, W. Liu, P. Dayal, D.H. Reneker, T. Kyu, Dynamics of hollow nanofiber formation during solidification subjected to solvent evaporation, Macromol. Theory Simulations. 15 (2006) 87-93. doi:10.1002/mats.200500034.

[49] J. Pelipenko, P. Kocbek, B. Govedarica, R. Rošic, S. Baumgartner, J. Kristl, The topography of electrospun nanofibers and its impact on the growth and mobility of keratinocytes, Eur. J. Pharm. Biopharm. 84 (2013) 401-411. doi:10.1016/j.ejpb.2012.09.009.

[50] R.Y. Basha, T.S. Sampath Kumar, M. Doble, Electrospun nanofibers of Curdlan ( $\beta-1,3$ glucan) blend as a potential skin scaffold material, Macromol. Mater. Eng. 302 (2017) 1600417. doi:10.1002/mame.201600417.

[51] A. Choromanska, J. Kulbacka, J. Harasym, R. Oledzki, A. Szewczyk, J. Saczko, Highand low-molecular weight oat beta-glucan reveals antitumor activity in human epithelial lung cancer, Pathol. Oncol. Res. (2017) 1-10. doi:10.1007/s12253-017-02783.

[52] L. Chen, R. Mirza, Y. Kwon, L.A. DiPietro, T.J. Koh, The murine excisional wound model: Contraction revisited, Wound Repair Regen. 23 (2015) 874-877. doi:10.1111/wrr.12338.

[53] C.D. Hinman, H. Maibach, Effect of air exposure and occlusion on experimental human skin wounds, Nature. 200 (1963) 377-378.

[54] M. Liu, F. Luo, C. Ding, S. Albeituni, X. Hu, Y. Ma, Y. Cai, L. McNally, M.A. Sanders, D. Jain, G. Kloecker, M. Bousamra, H. Zhang, R.M. Higashi, A.N. Lane, T.W.-M. Fan, J. Yan, Dectin-1 activation by a natural product $\beta$-glucan converts immunosuppressive macrophages into an M1-like phenotype, J. Immunol. 196 (2016) 3968-3968. doi:10.4049/jimmunol.1600345.

[55] T.F. de Almeida, T. de Castro Pires, A. Monte-Alto-Costa, Blockade of glucocorticoid 
receptors improves cutaneous wound healing in stressed mice., Exp. Biol. Med. (Maywood). 241 (2016) 353-8. doi:10.1177/1535370215612940.

[56] V.W. Wong, M. Sorkin, J.P. Glotzbach, M.T. Longaker, G.C. Gurtner, Surgical approaches to create murine models of human wound healing, J. Biomed. Biotechnol. 2011 (2011) 1-8. doi:10.1155/2011/969618.

[57] J.S. Choi, K.W. Leong, H.S. Yoo, In vivo wound healing of diabetic ulcers using electrospun nanofibers immobilized with human epidermal growth factor (EGF), Biomaterials. 29 (2008) 587-596. doi:10.1016/j.biomaterials.2007.10.012.

[58] H.S. Kim, H.S. Yoo, MMPs-responsive release of DNA from electrospun nanofibrous matrix for local gene therapy: In vitro and in vivo evaluation, J. Control. Release. 145 (2010) 264-271. doi:10.1016/j.jconrel.2010.03.006.

[59] R.-H. Dong, Y.-X. Jia, C.-C. Qin, L. Zhan, X. Yan, L. Cui, Y. Zhou, X. Jiang, Y.-Z. Long, In situ deposition of a personalized nanofibrous dressing via a handy electrospinning device for skin wound care, Nanoscale. 8 (2016) 3482-3488. doi:10.1039/C5NR08367B.

[60] M.R. Mohammadi, S. Rabbani, S.H. Bahrami, M.T. Joghataei, F. Moayer, Antibacterial performance and in vivo diabetic wound healing of curcumin loaded gum tragacanth/poly( $\varepsilon$-caprolactone) electrospun nanofibers, Mater. Sci. Eng. C. 69 (2016) 1183-1191. doi:10.1016/j.msec.2016.08.032.

[61] M. Naseri-Nosar, S. Farzamfar, H. Sahrapeyma, S. Ghorbani, F. Bastami, A. Vaez, M. Salehi, Cerium oxide nanoparticle-containing poly ( $\varepsilon$-caprolactone)/gelatin electrospun film as a potential wound dressing material: In vitro and in vivo evaluation, Mater. Sci. Eng. C. 81 (2017) 366-372. doi:10.1016/j.msec.2017.08.013.

[62] G. Ramanathan, T. Muthukumar, U. Tirichurapalli Sivagnanam, In vivo efficiency of the collagen coated nanofibrous scaffold and their effect on growth factors and proinflammatory cytokines in wound healing, Eur. J. Pharmacol. 814 (2017) 45-55. doi:10.1016/j.ejphar.2017.08.003.

[63] H.-L. Kim, J.-H. Lee, M.H. Lee, B.J. Kwon, J.-C. Park, Evaluation of electrospun $(1,3)-(1,6)-\beta$-D-glucans/biodegradable polymer as artificial skin for full-thickness wound healing, Tissue Eng. Part A. 18 (2012) 2315-2322. doi:10.1089/ten.tea.2011.0686.

[64] I. Noss, G. Doekes, P.S. Thorne, D.J. Heederik, I.M. Wouters, Comparison of the potency of a variety of $\beta$-glucans to induce cytokine production in human whole blood, Innate Immun. 19 (2013) 10-19. doi:10.1177/1753425912447129. 


\section{Supplement}

Nanofibers containing soluble beta-1,3/1,6-glucan improve wound healing in diabetic mice

Jostein Grip $^{\mathrm{a}, \mathrm{b}}$, Rolf Einar Engstad ${ }^{\mathrm{a}}$, Ingrid Skjæveland ${ }^{\mathrm{a}}$, Nataša Škalko-Basnet ${ }^{\mathrm{b}}$, Johan Isaksson $^{\mathrm{c}}$, Purusotam Basnet ${ }^{\mathrm{d}, \mathrm{e}}$, Ann Mari Holsæter, ${ }^{\mathrm{b}, *}$

${ }^{a}$ Biotec BetaGlucans AS, 9019 Tromsø, Norway

${ }^{\mathrm{b}}$ Drug Transport and Delivery Research Group, Department of Pharmacy, Faculty of Health Sciences, University of Tromsø The Arctic University of Norway, 9037 Tromsø, Norway

${ }^{\mathrm{c}}$ Drug Discovery and Design, Department of Chemistry, University of Tromsø The Arctic University of Norway, 9037 Tromsø, Norway

${ }^{\mathrm{d}}$ Women's Health and Perinatology Research Group, Department of Clinical Medicine, University of Tromsø The Arctic University of Norway, 9037 Tromsø, Norway

${ }^{\mathrm{e}}$ Department of Obstetrics and Gynecology, University Hospital of North Norway, Sykehusveien 5738, 9038 Tromsø, Norway.

\section{*Corresponding author}

Ann Mari Holsæter,

E-mail address: ann-mari.holsater@uit.no 


\section{Nanofiber swelling index}

The appearance of the two different nanofibers (the $\beta \mathrm{G}$ - and No $\beta \mathrm{G}$-containing fibers) upon soaking in the solution is presented in Sup. Figure 1; the $\beta$ G-containing nanofibers retained the original structure whereas the NoßG fibers appeared more affected by the exposure to aqueous medium. However, the swelling index indicated that the two nanofibers had a similar swelling capacity (Sup. Figure 1).

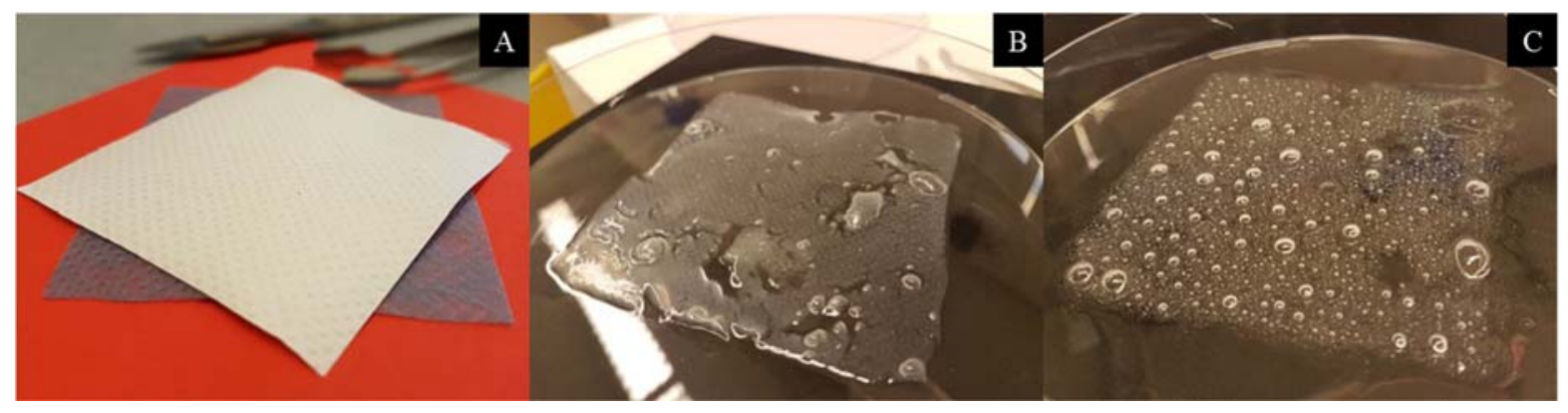

Sup. Figure 1. Photographs of the $8 \times 8 \mathrm{~cm}$ nanofibers sheets (A) before soaking in "Solution A" (B) soaked BG-containing nanofibers (C) soaked NoßG-containing nanofibers.

"Solution A" composing $142 \mathrm{mmol} \mathrm{Na}{ }^{2+}$ and $2.5 \mathrm{mmol} \mathrm{Ca}^{2+}$ in distilled water.

\section{Wound healing assessment}

During the in vivo wound healing study, pictures of the respective wounds (Sup. Figure ) were taken at different time points (Sup. Table 1), and the open wound area, wound contraction and re-epithelialization calculated using the computer Image Pro Plus software (version 4.1.0.0, Media Cybernetics, USA) 


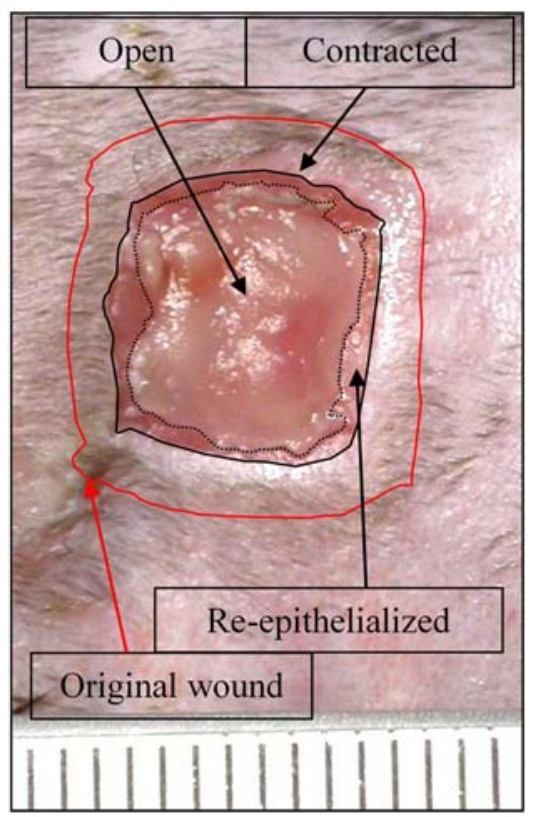

Sup. Figure 2. The wound healing parameters assessed by the Image Pro Plus software (version 4.1.0.0, Media Cybernetics, USA) to evaluate the progression of wound healing.

Sup. Table 1. Experimental setup for the wound healing experiments

\begin{tabular}{|c|c|c|c|c|c|c|c|c|c|c|c|c|}
\hline \multirow{2}{*}{$\begin{array}{c}\text { Formulation } \\
\text { applied }\end{array}$} & \multicolumn{12}{|c|}{ Day of treatment (x); day of (re-)anaesthetization (A); day of photography $(\mathrm{P})$} \\
\hline & 0 & 1 & 2 & 3 & 4 & 5 & 6 & 8 & 12 & 16 & 20 & 24 \\
\hline Positive Control & $\mathrm{x} / \mathrm{A} / \mathrm{P}$ & $\mathrm{x}$ & $\mathrm{x}$ & $\mathrm{x}$ & $\mathrm{x} / \mathrm{A} / \mathrm{P}$ & $\mathrm{x}$ & $\mathrm{X}$ & $\mathrm{A} / \mathrm{P}$ & $\mathrm{A} / \mathrm{P}$ & $\mathrm{A} / \mathrm{P}$ & $\mathrm{A} / \mathrm{P}$ & $\mathrm{P}$ \\
\hline Low $\beta G$ & $\mathrm{x} / \mathrm{A} / \mathrm{P}$ & & & & $\mathrm{x} / \mathrm{A} / \mathrm{P}$ & & & $\mathrm{x} / \mathrm{A} / \mathrm{P}$ & $\mathrm{A} / \mathrm{P}$ & $\mathrm{A} / \mathrm{P}$ & $\mathrm{A} / \mathrm{P}$ & $\mathrm{P}$ \\
\hline Medium $\beta G$ & $\mathrm{x} / \mathrm{A} / \mathrm{P}$ & & & & $\mathrm{X} / \mathrm{A} / \mathrm{P}$ & & & $\mathrm{x} / \mathrm{A} / \mathrm{P}$ & $\mathrm{A} / \mathrm{P}$ & $\mathrm{A} / \mathrm{P}$ & $\mathrm{A} / \mathrm{P}$ & $\mathrm{P}$ \\
\hline High $\beta G$ & $\mathrm{x} / \mathrm{A} / \mathrm{P}$ & & & & $\mathrm{x} / \mathrm{A} / \mathrm{P}$ & & & $\mathrm{x} / \mathrm{A} / \mathrm{P}$ & $\mathrm{A} / \mathrm{P}$ & $\mathrm{A} / \mathrm{P}$ & $\mathrm{A} / \mathrm{P}$ & $\mathrm{P}$ \\
\hline No $\beta G$ & $\mathrm{x} / \mathrm{A} / \mathrm{P}$ & & & & $\mathrm{X} / \mathrm{A} / \mathrm{P}$ & & & $\mathrm{x} / \mathrm{A} / \mathrm{P}$ & $\mathrm{A} / \mathrm{P}$ & $\mathrm{A} / \mathrm{P}$ & $\mathrm{A} / \mathrm{P}$ & $\mathrm{P}$ \\
\hline Negative Control & $\mathrm{x} / \mathrm{A} / \mathrm{P}$ & & $\mathrm{x}$ & & $\mathrm{X} / \mathrm{A} / \mathrm{P}$ & & $\mathrm{x}$ & $\mathrm{x} / \mathrm{A} / \mathrm{P}$ & $\mathrm{A} / \mathrm{P}$ & $\mathrm{A} / \mathrm{P}$ & $\mathrm{A} / \mathrm{P}$ & $\mathrm{P}$ \\
\hline
\end{tabular}

Positive Control: Hydrogel containing $10 \mu \mathrm{g}$ platelet-derived growth factor and $1 \mu \mathrm{g}$ transforming growth factor alpha, Low $\beta \mathrm{G}$ : Nanofiber containing $185 \mu \mathrm{g} \beta \mathrm{G}$, Medium $\beta \mathrm{G}$ : Nanofiber containing $360 \mu \mathrm{g} \beta \mathrm{G}$, High $\beta \mathrm{G}$ : Nanofiber containing $965 \mu \mathrm{g} \beta \mathrm{G}$, No $\beta \mathrm{G}$ : Nanofiber without $\beta G$, Negative Control: Water for injection

The in vivo experiment comprised animals (10 per group) treated with 6 different treatments. The representative photographs of the wound during the 24 days healing process are presented in Sup. Figure 3. 


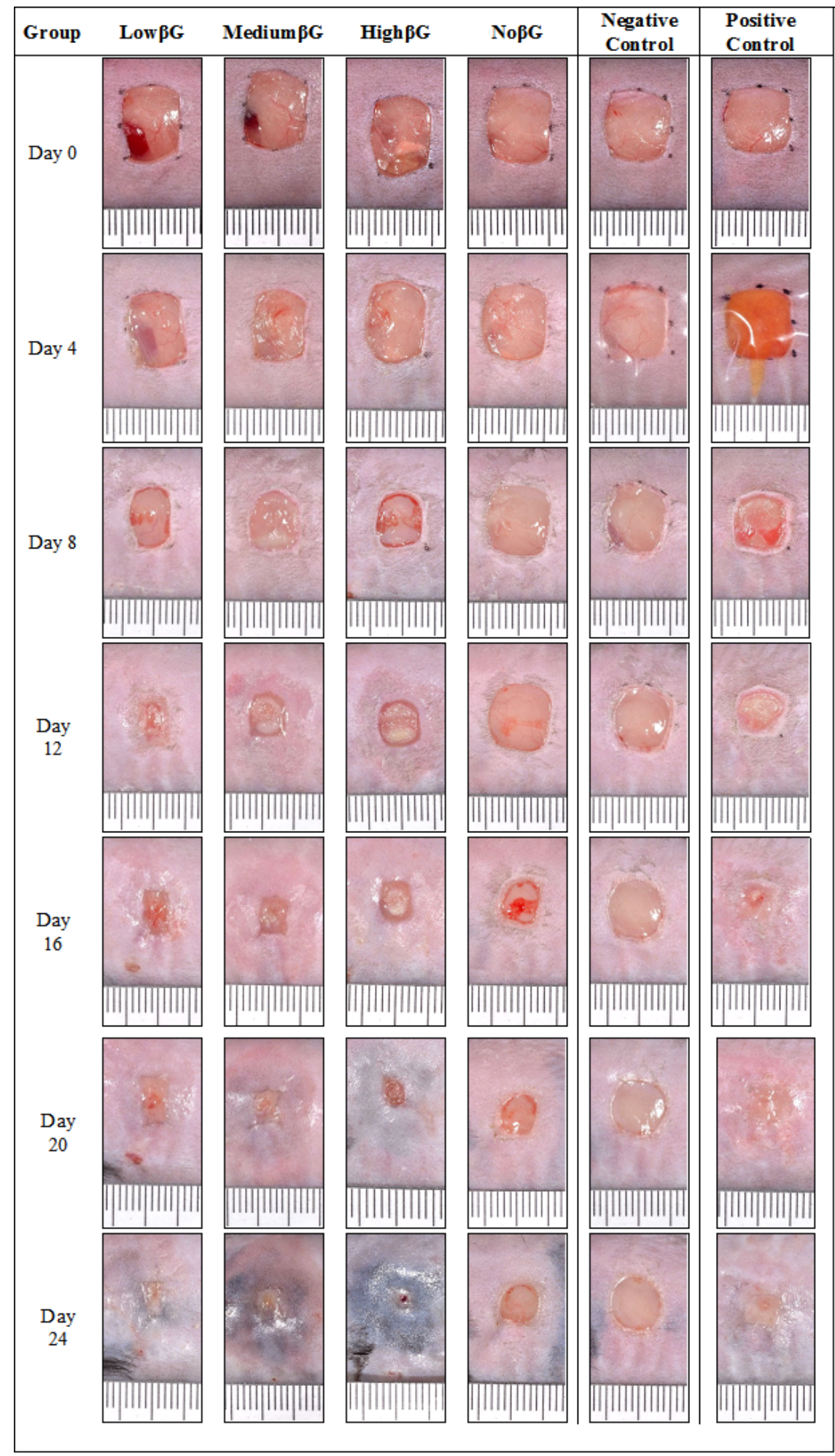

Sup. Figure 3. Photographs at different time points of animals from the different treatment groups;

Low $\beta G$ : Nanofiber containing $185 \mu \mathrm{g} \beta \mathrm{G}$, Medium $\beta \mathrm{G}$ : Nanofiber containing $360 \mu \mathrm{g} \beta \mathrm{G}$, High $\beta$ G: Nanofiber containing $965 \mu \mathrm{g} \beta \mathrm{G}$, No $\beta \mathrm{G}$ : Nanofiber without $\beta \mathrm{G}$, Negative Control: Water for injection, Positive Control: Hydrogel containing $10 \mu \mathrm{g}$ platelet-derived growth factor and $1 \mu \mathrm{g}$ transforming growth factor alpha. 